\title{
Perceived Impact of Non-contact Boxing on Daily Life and Occupational Participation at Home Among Individuals with Parkinson's Disease
}

\author{
Kristen E. Bignal \\ University of St. Augustine for Health Sciences \\ DOI: https://doi.org/10.46409/sr.PBWK9620
}

Follow this and additional works at: https://soar.usa.edu/capstones

Part of the Cognitive Psychology Commons, Exercise Science Commons, Neurology Commons, and the Occupational Therapy Commons

\section{Recommended Citation}

Bignal, K. E. (2020). Perceived Impact of Non-contact Boxing on Daily Life and Occupational Participation at Home Among Individuals with Parkinson's Disease. [Doctoral project, University of St Augustine for Health Sciences]. SOAR @ USA: Student Capstone Projects Collection. https://doi.org/10.46409/ sr.PBWK9620 


\title{
PERCEIVED IMPACT OF NON-CONTACT BOXING ON DAILY LIFE AND OCCUPATIONAL PARTICIPATION AT HOME AMONG INDIVIDUALS WITH PARKINSON'S DISEASE \\ by \\ Kristen E. Bignal
}

\begin{abstract}
A Capstone Presented in Partial Fulfillment of the Requirement for the Degree of DOCTOR OF OCCUPATIONAL THERAPY University of St. Augustine for Health Sciences
\end{abstract} April, 2020 


\section{PERCEIVED IMPACT OF NON-CONTACT BOXING ON DAILY LIFE AND OCCUPATIONAL PARTICIPATION AT HOME AMONG INDIVIDUALS WITH \\ PARKINSON'S DISEASE}

by

Kristen E. Bignal

has been approved

April, 2020

APPROVED:

Susan MacDermott, OTD, OTR/L, Doctoral Coordinator

Becki Cohill, OTD, OTR/L, Assistant Professor

Erin Schwier, EdD, OTD, OTR/L, Program Director

ACCEPTED AND SIGNED:

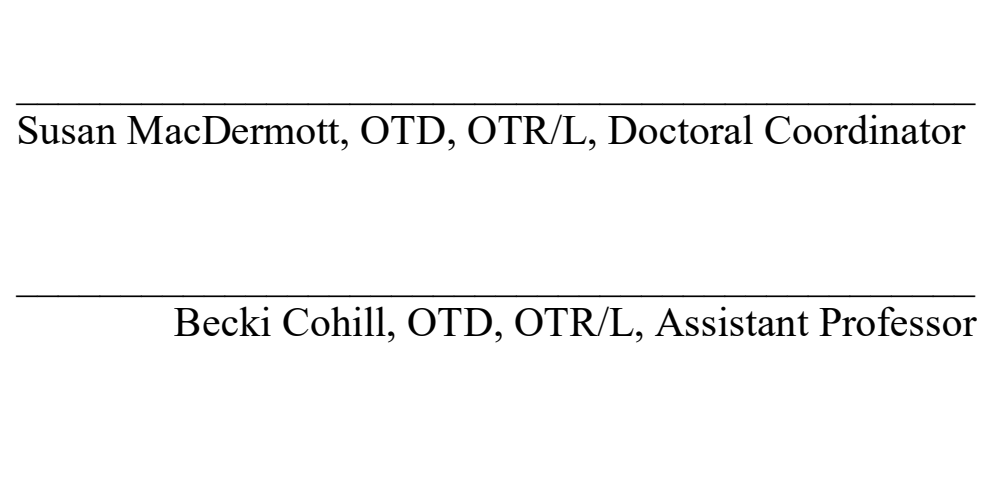

Erin Schwier, EdD, OTD, OTR/L, Program Director 


\section{Table of Contents}

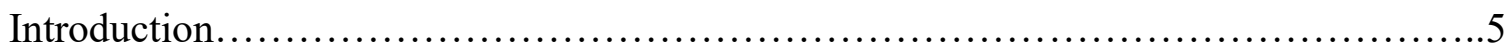

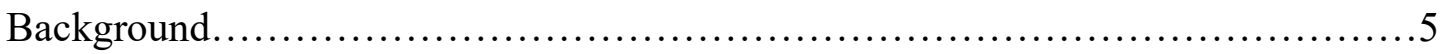

Statement of the Problem..................................................

Purpose Statement.........................................................

Rationale for Project................................................... 8

Significance of the Project............................................... 9

Project Objectives...................................................... 9

Assumptions, Limitations, and Delimitations...............................10

Literature Review.......................................................11

Occupational Therapy's Role.............................................11

Exercise-Related Programs.............................................13

Barriers to Occupational Participation........................................14

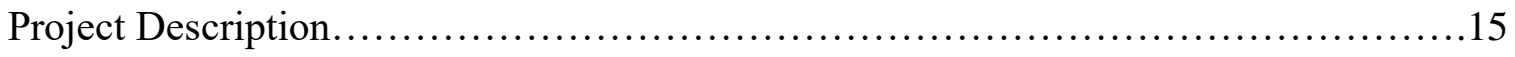

Timeline.............................................................

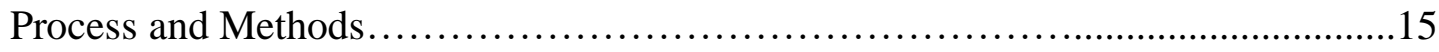

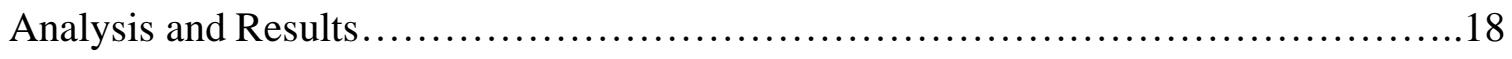

Analysis................................................................... 18

Results............................................................23

Discussion and Conclusion................................................ 32

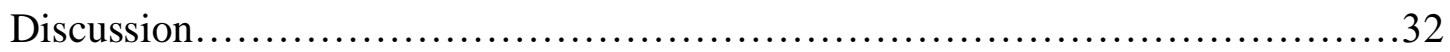

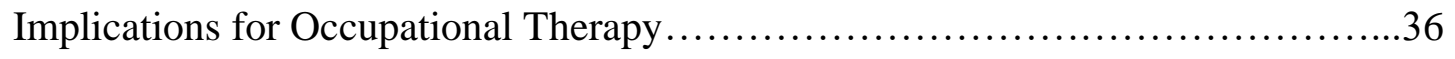

Limitations and Future Considerations.......................................... 37 


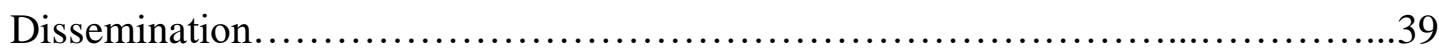

Conclusion.............................................................. 40

Acknowledgements.................................................40

References................................................................... 41

Appendix A - IRB Exempt Approval Letter..................................48

Appendix B - Recruitment Script.............................................49

Appendix C - Recruitment Flyer..........................................5 50

Appendix D - Consent Form............................................ 51

Appendix E - Semi-Structured Interview .......................................54

Appendix F - Grant Approval Letter.........................................55

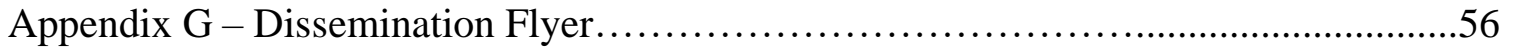

Appendix $\mathrm{H}$ - PowerPoint Presentation.....................................5 57

Appendix I - Research Poster...............................................64

Appendix I - Journal Publication...........................................65 


\section{Perceived Impact of Non-contact Boxing on Daily Life and Occupational Participatoin at Home Among Individuals with Parkinson's Disease}

\section{Background}

Parkinson's disease is the second most common neurodegenerative condition affecting nearly a million people annually in the United States, with 60,000 new cases each year (Fleisher et al., 2018). Parkinson's disease is characterized as the progressive loss of motor skills, balance, speech, and sensory function due to damaged, degenerative, or dead dopamine producing cells (Son \& Choi, 2018). Motor symptoms of Parkinson's disease include tremor, rigidity, bradykinesia (slowed movements), festinating gait (short, shuffling steps), and a decline in strength, balance, and secondary cardiovascular fitness (Son \& Choi, 2018). Non-motor complications include fatigue, apathy, sleep disturbances, depression, and cognitive decline (Uhrbrand et al., 2015). Both motor and non-motor limitations compromise safety and satisfaction with participation in meaningful daily occupations (Serrano-Dueñas et al., 2018). Daily occupations are defined by the American Occupational Therapy Association (AOTA) as "the things that people do that occupy their time and attention; meaningful, purposeful activity; the personal activities that individuals choose or need to engage in and the ways in which each individual actually experiences them" (AOTA, 2014, p. 1237).

The progression of Parkinson's disease is associated with loss of independence in activities of daily living (ADLs), instrumental activities of daily living (IADLs), and many other meaningful occupations (Serrano-Dueñas et al., 2018). ADLs are activities concerning taking care of one's own body (e.g., dressing, eating, transferring in and out of bed); IADLs are required activities that support daily living within the home and 
community. In comparison to ADLs, IADLs such as housework and shopping often require more involvement physically and cognitively (AOTA, 2014). A cross-sectional study conducted in 2014 utilized the Performance Assessment of Self-Care Skills (PASS) to measure independence with IADLs among individuals with relatively early and mild Parkinson's disease (Foster, 2014). The PASS is an objective, criterion-referenced measure of activity performance (Foster, 2014). Nine cognitively demanding IADLs were administered for this study (Foster, 2014). The Parkinson's disease group demonstrated greater deficits and lower independence summary scores in comparison to the nonParkinson's disease group (Foster, 2014). These results are relevant because IADLs are critical for productive and independent living. Strategies to support IADL performance and other complex occupations in the early stages of Parkinson's disease may allow individuals to prolong their independence in participation in daily activities (Foster, 2014).

The Occupational Therapy Practice Framework: Domain and Process, $3^{\text {rd }}$ Edition (The Framework) is an official document of the AOTA (2014). This document outlines domains and processes which describe occupational therapy practice (AOTA, 2014). The Framework defines occupational therapy as "the therapeutic use of everyday life activities (occupations) with individuals or groups for the purpose of enhancing or enabling participation in roles, habits, and routines in the home, school, workplace, community, and other settings" (AOTA, 2014, p S1). This document identifies and outlines a range of occupations including ADLs, IADLs, rest and sleep, leisure, and social participation (AOTA, 2014). 
A number of community programs and organizations offer exercise classes to alleviate symptoms of Parkinson's disease and sustain quality of life (Combs et al., 2013). Regular exercise leads to a feeling of physical and emotional well-being (Amara \& Memon, 2018). Exercise can alleviate some secondary orthopedic effects of rigidity such as shoulder, hip, and back pain, and improve motor and non-motor symptoms (Amara \& Memon, 2018; Shulman et. al., 2013; Suchowersky et al., 2006). This study will focus on non-contact boxing as the form of exercise of interest and will recruit from a program called "Rock Steady Boxing" that is specifically aimed to combat the onset or progression of Parkinson's disease symptoms (Combs et al., 2011). The non-contact boxing classes use an interval training format that combine aerobic, resistance, and flexibility exercises. Four levels of classes are offered depending on the participant's level of functioning.

\section{Statement of the Problem}

The literature appears to omit assessment of occupational participation in the home environment among individuals with Parkinson's disease that participate in group exercise classes. A greater understanding of how the impact of non-contact boxing translates to the home environment is needed to promote occupational participation in the home and identify areas for occupational therapists to address directly through services or by consulting exercise programs.

\section{Purpose Statement}

This capstone project aims to determine whether individuals with Parkinson's disease perceive an impact on their daily life and occupational participation secondary to participating in non-contact boxing. This will be addressed through a research study by 
utilizing qualitative measures to fully understand participants' experiences and impact on everyday life at home.

\section{Rationale for Project}

The Person Environment Occupational Performance (PEOP) model, developed by Baum and Christiansen, categorizes performance of necessary and valued occupations into four pieces: the person (i.e., intrinsic factors), the environment (i.e., extrinsic factors), the occupation (i.e., task at hand), and performance (i.e., the person's ability to perform said occupation) (Smith \& Hudson, 2012). Intrinsic factors include physiological, cognitive, spiritual, neuro-behavioral, and psychological elements; extrinsic factors include social support, culture and values, built environment and technology, and the natural environment (Smith \& Hudson, 2012). The PEOP model will be used as a template to address occupational performance concerns of individuals with Parkinson's disease.

The PEOP model guides the analysis of factors necessary for occupational performance and participation. Non-contact boxing classes focus on many factors such as muscle stiffness, posture, eye-hand coordination, balance, socialization, and reaction time (Laliberte, 2017). However, there are many additional factors from the PEOP model to consider to promote performance and participation in daily occupations in the home (Smith \& Hudson, 2012). For example, the environmental shift from the boxing gym to home creates contextual barriers that may hinder safety and participation in desired activities. Timing is another environmental factor to address. The participants' abilities within the hours after class may look vastly different compared to a day they do not 
participate in a boxing class (Combs et al., 2011). Additionally, social supports and psychological elements are just as important to consider (Sheehy et al., 2017).

\section{Significance of the Project}

This capstone project aims to determine participants' perceptions of how noncontact boxing impacts their daily lives and participation in meaningful occupations at home. Semi-structured interviews and the Parkinson's Disease Questionnaire (PDQ-39) will be used to collect information from participants. This information will be organized into common themes of how participants believe this form of exercise treatment carries

over from the gym to their home environment. Health professionals will then be aware of the clients' perceived needs and barriers to participation.

\section{Project Objectives}

1. Assess needs of individuals with Parkinson's disease through research and literature review

2. Observe and participate in the non-contact boxing classes through the Rock Steady Boxing Headquarters gym in Indianapolis, IN

3. Recruit participants and organize interview schedule

4. Complete semi-structured interview with participants at the Rock Steady Boxing Headquarters gym in Indianapolis, IN

5. Transcribe and code data

6. Develop themes and summarize results

7. Provide an educational session for non-contact boxing participants and family members on results of project to promote occupational performance 


\section{Assumptions, Limitations, and Delimitations}

\section{Assumptions}

The current literature strongly suggests that Parkinson's disease presents barriers to occupational participation and impacts one's performance patterns (Sperens et al., 2018). Additionally, individuals in advanced stages of the disease tend to have greater performance deficits and barriers to participation in meaningful occupations (Sperens et al., 2018). This capstone project assumes that there may be carryover of benefits secondary to boxing from the gym to the home environment. It is assumed that participants from this capstone project represent the greater population of non-contact boxing participants.

\section{Limitations}

The provided length of time for this capstone project is acknowledged as a limitation. Additionally, the number of participants willing to participate is uncontrollable and a smaller sample size may limit the ability to generalize results to the larger population of all non-contact boxing participants. Since participants will be asked to share their perceptions of the impact of non-contact boxing on their home occupations, their opinions will be recorded rather than having obtained an objective measure from a licensed therapist. The goal of this study is to determine participants' perceptions, rather than a factual measure of their actual performance.

\section{Delimitations}

This project solely focuses on the non-contact boxing participants that train in Indianapolis, Indiana at a Rock Steady Boxing gym on Hillsdale Court. Parkinson's disease presents differently and progresses at different rates in each individual (Fleisher 
et al., 2018). This study intends to include and represent all stages of Parkinson's disease. The study aims to concentrate on the participants' perception of their participation in the home environment. Due to the specific focus of occupations in the home, this study limits assessment of occupations in the community environment.

\section{Literature Review}

This literature review explores three main themes identified in evidence-based research to support this study: occupational therapy's role in the treatment of Parkinson's disease, current exercise-related programs for individuals with Parkinson's disease, and barriers to occupational participation among those with Parkinson's disease.

\section{Occupational Therapy's Role}

Parkinson's disease presents physical, psychological, social, and functional barriers to everyday life (Son \& Choi, 2018). Well-known symptoms of Parkinson's disease include bradykinesia, muscular rigidity, and a resting tremor (Morberg et al., 2014). Individuals with Parkinson's disease also experience many non-motor symptoms including depression, cognitive impairments, fatigue, and sleep disorders. For individuals with Parkinson's, non-motor symptoms may have a greater impact on quality of life than motor symptoms (Morberg et al., 2014; Serrano-Dueñas et al., 2018). Currently, occupational therapy and other rehabilitation professionals target these motor and nonmotor symptoms with three main intervention strategies: exercise, environmental cues/stimuli, and cognitive-behavioral strategies (Foster et al., 2014).

Individuals with Parkinson's disease maintain their quality of life best by prioritizing valued occupations during occupational therapy interventions (Sperens et al., 2018). Interventions may follow an acquisitional, restorative, or adaptive approach 
depending on the stage of the disease (Jansa \& Aragon, 2015). Restorative occupational therapy promotes smooth initiation and execution of movements and focuses on addressing ADLs, IADLs, leisure, and work-related tasks (Shah \& Nolen, 2006). Occupational therapists recognize that a cognitively challenging random practice environment is better generalized to ADL and IADL tasks rather than blocked practice (Sidaway et al., 2016). Additionally, intense functional task training, within the capabilities of the client, has a positive effect on ADL performance (Perry et al., 2019). Home-based occupational therapy allows patients with Parkinson's disease to have improved self-perception and confidence when performing daily activities because they are more comfortable in their natural environment (Sturkenboom et al., 2014). One common and well-researched intervention approach is Lee Silverman Voice Treatment BIG (LSVT-BIG); this behavioral treatment approach aims to increase movement amplitude across the limbs and may be completed in home, clinic, or community settings (Fox et al., 2012). "BIG" refers to the emphasis on increasing size of movements as people living with Parkinson's disease tend to move differently, with gestures and actions that become smaller and slower (LSVT Global, 2020).

Currently, rehabilitation for individuals with Parkinson's disease is typically focused on physical outcomes and symptom management (Vaartio-Rajalin et al., 2019). With client-centered interventions, positive cognitive and psychosocial health are often secondary outcomes (Vaartio-Rajalin et al., 2019). Furthermore, research shows that physical rehabilitation fosters social bonding and promotes social participation (Lizon et al., 2017). 


\section{Exercise-Related Programs}

Academic research discusses many exercise-related community programs for individuals with Parkinson's disease including: LSVT-BIG, intensive exercise therapy, dance therapy, meditation-based exercise, yoga, and boxing (Combs et al., 2011; Ebersbach et al., 2010; Kwok et al., 2017; Son \& Choi, 2018; Toygar et al., 2018; Uhrbrand et al., 2015). All exercise modalities besides boxing will be discussed first, with a more in-depth review of the effects of boxing to follow. LSVT-BIG was introduced as a behavioral treatment approach focusing on big, exaggerated movements to increase movement amplitude across the limbs (Ebersbach et al., 2010). Intensive exercise therapy includes resistance training, endurance training, and other intensive training modalities (Uhrbrand et al., 2015). Intensive exercise therapy has positive effects on muscle strength, balance, walking, and cardio-respiratory fitness (Uhrbrand et al., 2015). Morberg and colleagues (2014) suggest that intensive exercise may influence nonmotor symptoms as well. Dance therapy provides good aerobic exercise, and also improves strength, flexibility, focus, and cardiovascular health (Toygar et al., 2018). Dance is beneficial for fall prevention and social participation as well (Toygar et al., 2018). Mindfulness meditation-based exercise programs are associated with improved anxiety, depression, sleep, cognitive functions, and quality of life (Son \& Choi, 2018). Similarly, yoga decreases psychological distress and improves overall well-being (Kwok et al., 2017).

Evidence-based research on the benefits of boxing to treat symptoms of Parkinson's disease provides the framework for this project. Individuals that participate in boxing training demonstrate short-term and long-term improvements in balance, gait, 
ADLs, and quality of life (Combs et al., 2013). Another study specifically looked at the effects of boxing on social supports and challenges. This study found that participation in a group boxing program provides relief from anxiety and depression and strengthens feelings of inspiration and support (Sheehy et al., 2017). Laliberte (2017) states that emotional empowerment might be the most important factor to help manage symptoms of Parkinson's disease.

\section{Barriers to Occupational Participation}

The progression of Parkinson's disease is associated with loss of independence in ADLs (e.g., dressing, eating, transferring in and out of bed), IADLs (e.g., housework and shopping), and many other meaningful occupations (Perry et al., 2019). Multidisciplinary care teams aim to maximize functional levels and independence of individuals with Parkinson's disease when performing occupations (Fleisher et al., 2018; Miertová et al., 2014). One study found that an individual's manual hand dexterity positively correlates to ADLs, meaning that manual hand dexterity is an important factor for predicting physical performance in daily occupations among those with Parkinson's disease (Choi et al., 2017). Furthermore, individuals with early and mild Parkinson's disease without dementia demonstrated measurable deficits when performing cognitively demanding IADLS (e.g., meal prep with oven/stove, paying and mailing bills, shopping, medication management, small home repairs) (Foster, 2014).

Psychosocial factors such as depression, cognitive impairments (e.g., dementia and language difficulties), and a lack of self-purpose have a significant impact on how individuals with Parkinson's disease view their quality of life (Rosińczuk \& Kołtuniuk, 2017). Additionally, deficits in language such as verbal, nonverbal, comprehension, and 
fluency are common symptoms of Parkinson's disease which may impact many important occupations including social engagements such as participating in conversation at mealtime and communicating basic wants or needs (Wolff \& Benge, 2019).

\section{Project Description}

\section{Timeline}

Approval from the University of St. Augustine for Health Sciences Institutional Review Board (IRB) committee was granted for this study before the initiation of the capstone experience. The IRB is a constituted group formally designated to review and monitor research to protect the rights of human research subjects (University of St. Augustine for Health Sciences, 2020). See Appendix A for the approval letter. The capstone experience consisted of 16 weeks from November 2019 - April 2020. The student researcher spent the first 3 weeks observing classes and weeks 4-8 recruiting participants and completing interviews. Weeks 9-16 involved coding and analyzing data and reporting thematic analysis in this written report. Results were organized and dissemination occurred at the end of the capstone project in April 2020.

\section{Process and Methods}

All participants, regardless of age or gender, were welcome to participate. Prior to recruitment, the student researcher observed and participated in boxing classes for several weeks to build rapport with clients and develop a greater understanding of the boxing program. Participant observation is a technique used in qualitative research where the researcher becomes an active participant in the activities of the group that is being studied (Portney \& Watkins, 2009). This observation period yielded a level of connection between the student researcher and the participants by allowing the student researcher to 
better understand the participants' experiences with non-contact boxing. After completion of the observation period and at the end of the boxing classes, the student researcher read the recruitment script (see Appendix B) to the participants and asked for anyone interested to retrieve a copy of the recruitment flyer (see Appendix C) from the front desk. The method of convenience sampling involves participant selection based on availability (Portney \& Watkins, 2009). A pre-determined goal of 20 participants (i.e., five from each level of class offered at the non-contact boxing gym) was set. A sample size of 20 participants is adequately large to identify common themes while remaining small enough to ensure that ample resources are available for in depth analysis (Sheehy et al., 2017).

Seventeen participants $(n=17)$ from the Rock Steady Boxing Headquarters gym in Indianapolis, Indiana participated in this study, three participants less than the planned number. The four class levels offered at Rock Steady Boxing (i.e., PD1, PD2, PD3, PD4) are roughly associated with the stage of Parkinson's disease outlined by the Parkinson's disease foundation (Parkinson's Foundation, 2020). Only one level four participant volunteered for the study. Figure 1 displays the number of participants in each class level. Age range of participants was 44-87. Gender breakdown of the 17 participants includes three females and 14 males. Two participants reported full-time employment, two participants reported part-time employment, and 13 reported retirement. Participants reported being diagnosed with Parkinson's disease for $<1$ to 16 years and have been participating in non-contact boxing for $<1$ to 13 years. The most recent participant to join Rock Steady Boxing started in October 2019. 


\section{Figure 1}

Number of Participants by Level

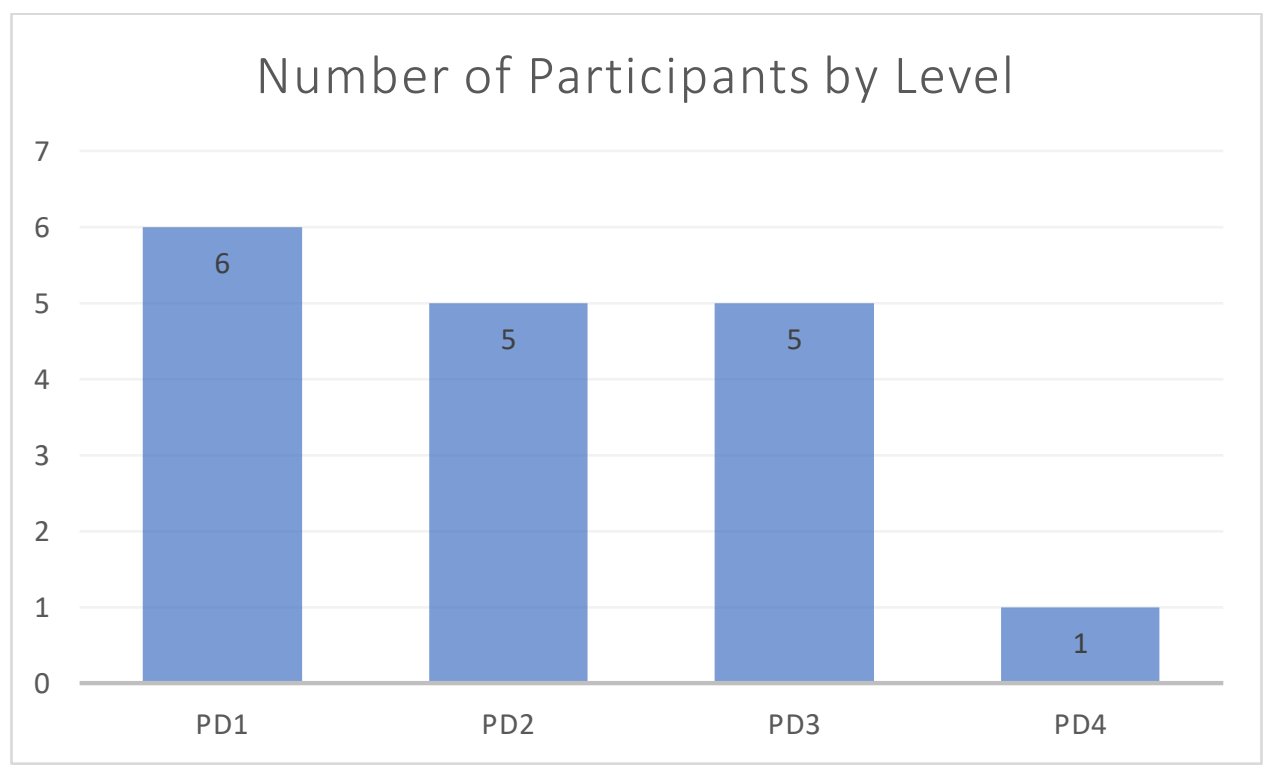

The boxers signed up for a one-hour time slot to participate in a semi-structured interview. Prior to interviews, participants were asked to provide consent (see Appendix D) to allow the interview to be audio recorded. All participants $(n=17)$ provided their verbal consent. The interview took place in a quiet room at the boxing gym. Face-to-face interviews are more effective for establishing rapport between the student researcher and the participant; this personal interaction helps elicit upfront and honest responses (Portney \& Watkins, 2009). The semi-structured interview (see Appendix E) included open-ended questions about the participants' home occupations and their perceptions of how the non-contact boxing class has impacted their participation in these meaningful occupations. Interview questions were developed to outline ADLs and IADLs from The Occupational Therapy Practice Framework $(O T P F)$ and influenced by two recent studies that collected information using semi-structured interviews ("Occupational Therapy Practice Framework,” 2017; Sheehy et al., 2017; Sperens et al., 2018). A semi-structured 
interview script guided discussion and provided a basis for comparing responses when the data was later coded to identify themes (Portney \& Watkins, 2009). The script was flexible enough to allow the interviewer to expand and ask follow-up questions relevant to a participant's specific circumstances (Portney \& Watkins, 2009). The researcher used best judgement to skip or reword certain questions as appropriate for each individual participant. If needed for communication purposes or participant comfort, a family member or caregiver was welcomed to sit in during the interview. It is understood that a family member or caregiver may present a different perspective than the individual with Parkinson's disease.

The researcher acknowledges this study originally compromised mixed methods including an interview and PDQ-38 as outcome measures. The researcher made a clinical decision to not follow through with the PDQ-39, as the information provided from the questionnaire did not directly provide information on how non-contact boxing impacted their everyday life, but rather general questions on their health and well-being unattached to the non-contact boxing classes. The interviews proved a more valuable use of participants' time rather than the completion of a questionnaire. The researcher continued research as a qualitative study with the interview as the sole outcome measure.

\section{Analysis and Results}

\section{Analysis}

Seventeen interviews were completed, audio recorded, and transcribed. The nature of a semi-structured interview provided the opportunity for in-depth analysis of the participants' responses (Portney \& Watkins, 2009). The researcher used Temi, advanced speech recognition software, to significantly improve and accelerate the transcription 
process allowing for a more comprehensive analysis (“Temi”, 2020). The researcher reread the transcribed interviews while listening to the audio recordings to correct the generated transcription to ensure accuracy. The researcher developed an in-depth understanding of responses through this process. A second web-based application, Dedoose version 8.3.17, was used to analyze the qualitative data (2020). The researcher attended a free introductory webinar on Dedoose and utilized videos on the website to become familiar with the platform. The researcher also received training from a qualitative research expert on use of the software. A grant to cover expenses for Temi was applied for and received through the university. See Appendix F for the approval letter. A free trial offered by Dedoose was used for thematic analysis.

The researcher used thematic analysis to analyze the qualitative data, guided by Braun and Clarke (2006). Thematic analysis is a method for identifying, analyzing, and reporting themes within data (2006). Braun and Clarke outline six phases of thematic analysis presented in Table 1 . 


\section{Table 1}

Braun and Clarke's Phases of Thematic Analysis

Phase
1. Familiarizing yourself

with your data

2. Generating initial codes

3. Searching for themes

4. Reviewing themes

5. Defining and naming themes

6. Producing the report
Description of the Process

Transcribing data (if necessary), reading and re-reading the data, noting down initial ideas.

Coding interesting features of the data in a systematic fashion across the entire data set, collating data relevant to each code.

Collating codes into potential themes, gathering all data relevant to each potential theme.

Checking in the themes work in relation to the coded extracts (Level 1) and the entire data set (Level 2), generating a thematic 'map' of the analysis.

Ongoing analysis to refine the specifics of each theme, and the overall story the analysis tells; generating clear definitions and names for each theme.

The final opportunity for analysis. Selection of vivid, compelling extract examples, final analysis of selected extracts, relating back of the analysis to the research question and literature, producing a scholarly report of the analysis.

Note. Adapted from "Using Thematic Analysis in Psychology," by V. Braun and V.

Clarke, 2006, Qualitative Research in Psychology, 3(2), p. 35

(http://dx.doi.org/10.1191/1478088706qp063oa).

Phase 1 was initiated by repeatedly and actively reading the data before beginning any coding. The researcher became familiar with the depth and breadth of the content by conducting interviews, listening to audio tape, actively participating in transcription, and reading through the transcripts multiple times. It is important that the researcher is immersed in the data, taking notes or marking ideas that will be helpful in phase 2 (Braun \& Clarke, 2006).

The researcher used an inductive or 'bottom up' approach to code data meaning equal attention was given to each data set to produce diverse codes (Braun \& Clarke, 2006). This process began phase 2 . The researcher initially coded an average of 46 codes per participant, ranging from 31-74 codes per participant. The researcher coded by 
highlighting, tagging, and naming selections of text within the data set. A code was marked if it appeared interesting to the researcher, regardless of whether it directly related to the research topic or not. Braun and Clarke (2006) recommend coding for as many potential themes or patterns as possible. The student researcher consulted with a doctoral supervisor and qualitative research expert to review codes prior to moving on to the next steps.

After initial coding was completed, the search for themes began (phase 3). It is important to recognize that the researcher played an active role in identifying themes and that themes do not just 'emerge' or become 'discovered' (Braun \& Clarke, 2006). A theme captures and represents a level of patterned responses (Braun \& Clarke, 2006). Braun and Clarke (2006) highlight the importance of flexibility and consistency when determining themes. The researcher chose to focus on prevalence of codes within each data set (i.e., one participant's interview) and across the entire data set (i.e., all 17 participant interviews) to identify themes. Salience of a coded excerpt was deemed just as important. As a variety of topics came up during interviews, themes generated had to answer the topic of this research study. The researcher used hand-written mind-maps and discussion with the second researcher to identify themes. Mind-maps are a visual representation technique used to sort and connect codes into different categories or groups (Braun \& Clarke, 2006). Main themes were developed after multiple revisits to review codes and interviews. Some initial codes became main themes, some formed subthemes, and some codes were grouped as “miscellaneous”, meaning they didn't fit into any of the themes or subthemes. Some "miscellaneous" themes provide discussion points to address in later sections of this paper. 
Phase 4 included reviewing the themes at the level of the coded data. This means that each data excerpt was reviewed and organized into one of the potential themes to develop a coherent pattern. This included organizing potential themes and codes into recognizable patterns or themes. Table 2 displays the code count for potential themes.

Table 2.

Code Count for Potential Themes

\begin{tabular}{|c|c|c|c|}
\hline \multirow{3}{*}{$\begin{array}{l}\text { Potential Theme } \\
\text { Psychological/Mental health }\end{array}$} & Code & Number of Codes & Total \\
\hline & $\begin{array}{l}\text { Anxiety } \\
\text { Depression } \\
\text { Confidence } \\
\text { Energy/drive } \\
\text { Negative mental health } \\
\text { Positive mental health } \\
\text { Stress }\end{array}$ & $\begin{array}{l}22 \\
8 \\
6 \\
7 \\
14 \\
13 \\
27 \\
3\end{array}$ & \\
\hline & & & 100 \\
\hline Community & $\begin{array}{l}\text { Motivation to continue } \\
\text { Motivation to join } \\
\text { Social support }\end{array}$ & $\begin{array}{l}13 \\
30 \\
12 \\
41\end{array}$ & \\
\hline & & & 96 \\
\hline Routines & $\begin{array}{l}\text { Morning routine } \\
\text { Evening routine } \\
\text { Lifestyle }\end{array}$ & $\begin{array}{l}13 \\
18 \\
4 \\
26\end{array}$ & 61 \\
\hline
\end{tabular}

This table helped the researcher organize potential themes and codes into themes and subthemes, respectively. This process involved reading through coded excerpts to find meaning behind codes. The student researcher consulted with a doctoral supervisor, qualitative research expert, and mentor to review potential themes. This process concluded with three overarching themes. Once satisfied, the researcher reported definitive themes organized in Table 3. 
Table 3.

Themes and Subthemes

\begin{tabular}{ll}
\hline Theme & Subthemes \\
\hline Psychological benefits & Increases confidence \\
& Boosts psychological health \\
\hline Sense of community & Feeling understood and supported \\
& Gateway to new social relationships \\
& Comradery \\
\hline Framework to develop routines & New routines \\
& Well-balanced lifestyle \\
\hline
\end{tabular}

Phase 5 includes defining and naming themes. Phase 6 includes the final analysis in a written report. Next, results will provide a description and evidence for each theme and subtheme.

\section{Results}

Final data analysis delivered three overarching themes and respective subthemes addressing the research topic, 'perceived impact of non-contact boxing on daily life and occupational participation at home among individuals with Parkinson's disease.' The three themes include: psychological benefits, sense of community, and framework to develop routines.

\section{Psychological Benefits}

Whether it related to confidence, attitude, stress, anxiety, or depression, a majority of the participants expressed that participation in non-contact boxing improved their psychological and mental health. This theme is separated into two sub-themes: increases confidence and boosts psychosocial health.

Increases Confidence. From the participant experiences and perceptions shared, non-contact boxing helps participants increase feelings of confidence which positively impacts their abilities to do everyday occupations. 
I think the fact that I had been going to Rock Steady boxing before I fell helped me as far as strengthening me and gaining confidence that I rehabbed better and my attitude was better after I fell... And so I was building myself back up as far as confidence and setting some goals (Participant 13).

When other participants were asked how non-contact boxing had impacted their ability to participate in different occupations, they too credited non-contact boxing with providing them confidence and other psychosocial benefits.

Right off hand, the best I can say is I may be improved from a strength and goal standpoint and belief in myself, which is important... So, from that standpoint there was some confidence and the perseverance, mental strength, I think that maybe I have that I wouldn't have had otherwise (Participant 14).

Participant 17 also remarked on their newfound confidence. "I think what it has done is given me confidence, especially knowing you're walking into friends it has held back the symptoms." Participants perceive their psychological confidence to directly impact their confidence in physical abilities too. “...I have a lot more confidence in my balance, in my strength than I did before Rock Steady. I can't imagine the difference really if I weren't in it" (Participant 3).

Boosts Psychological Health. Participants report decreased levels of anxiety and depression on a day to day basis since starting non-contact boxing classes as well as immediate relief of anxiety and depression after non-contact boxing classes compared to before. Participant 8 acknowledged that their biggest challenge is managing anxiety. When asked if non-contact boxing impacts their anxiety they responded, 
Well, I admit that every time we come to class, I'm a little nervous, but every time I leave class it's kind of gone away. So, it helps... That's why I think coming more frequently will not only help me physically but mentally (Participant 8).

Participants commonly stated non-contact boxing decreased common and negative symptoms of mental health and boosted psychological and physical health. Participant 1 stated, “...It's helped me to be stronger and have more energy... and definitely Rock Steady Boxing has helped me with my depression.” Many participants stated that their best days are the days they box. They said it provided them with more energy and made them feel better. Participant 6 exclaimed, "But if I go to Rock Steady... I'm good for the whole day. Energizes me. And like I said, then I forget that I'm having a bad day. It just clears up." No matter how the participants felt prior to boxing that day, they credited their participation in non-contact boxing for shifting their mindset.

Because I wake up some mornings thinking I do not want to go. And then I think what a gift it is to get yourself out of bed and get over there. And every time I finished class, I'm glad I did it. So that benefit, it's huge (Participant 17). Participant 1 shared what he finds to be the greatest benefit, "And I think what I get out of it the most is it helps my attitude and it keeps me positive." All of the participants used different words to share their thoughts on how non-contact boxing impacted their overall psychosocial health and how it carried over to their everyday life. Participant 16 said, "It helped me accept the disease, I guess. It's really been powerful from a psychological standpoint." Participants report experiencing a newfound view on their situation. Not only did non-contact boxing make them feel better temporarily but provided them with a 
fresh sense of hope and a new outlook inspiring each of them to move forward despite their challenges.

\section{Sense of Community}

Participants reported that the community atmosphere within the boxing gym was one that permeated into their everyday lives at home and within the community. This theme was separated into three sub-themes: feeling understood and supported, gateway into new social relationships, and comradery.

Feeling Understood and Supported. Participants reported feeling understood and supported by other boxers, coaches, and staff. The gym environment provided boxers with a safe place, somewhere they were welcome and not looked at differently for the challenges they face.

...It's great for my morale, you know, so it's a happy thing. It's great seeing the same people and it's great. Being able to talk about it with people who are suffering similar things. Yeah... That gives us a place where we can be normal, but I don't have to worry about whether anybody's going to notice that weird symptoms we have (Participant 6).

Participants stated the welcoming and inclusive environment allowed them to feel accepted despite the onset of symptoms. Participant 17 stated, "You don't have to explain. You can walk in the gym and say, I didn't sleep last night. And everybody goes, okay. Yeah, we know. Yeah.” Participant 12 specifically addressed feelings of understanding and support offered to loved ones as well. "I feel at home here, I feel like my friends are here. The people who are here understand me and the people who are here 
help me and help my wife know what to do" (Participant 12). Similarly, Participant 10 commented on the resources and people able to provide additional support when needed. I would say knowing that there are other people out there who are experiencing the same thing I am and that's a resource I can tap into, that is a strength that I can lean on, and it's my rock, keeps me steady (Participant 10).

Gateway to New Social Relationships. Boxing decreased social isolation in the home by providing social interaction through non-contact boxing classes, lunch dates, and phone calls. Participant 17 shared, "I frequently will have lunch with somebody during the week... and the community here is such that you can, I mean everybody's pretty low key so you could call and say, hey, can you meet me...?" Fellow boxers provided an outlet for social engagements and common ground for relationships to develop. The opportunity to make long-lasting friendships is a significant support stated by many. Many participants stated that their closest friendships were established at noncontact boxing classes. When asked why non-contact boxing means so much to them, Participant 9 shared, "Friendship. Knowing people. Having something to do, place to go. Just being around people.” New social relationships prevented participants from lonely isolation. Some participants relied mostly on family to enjoy social engagements with, but for others, this community is their family.

I think that without this community of people, I would probably feel really isolated. I think even though they're not with me and all the situations, I can think about the fact that I'm not the only person that I know that has to deal with it (Participant 4). 
Both physical and social environments provided support for these individuals. The built gym where participants gather to participate in group non-contact boxing classes construct the social environment that expands outside of the four walls into their homes and communities.

If I get a chance to get rid of my Parkinson's, but to give up my Parkinson's I had to lose my friends that I've got to be friends with, I would go ahead and keep my friends and keep my Parkinson's (Participant 12).

Comradery. An atmosphere of friendship and family provided participants with a sense of community and belonging while a spirit of 'tough-love', a commonly stated phrase at the Rock Steady Boxing gym, kept workouts upbeat and moods bright. Participant 5 shared, "I appreciate the comradery. Everybody has Parkinson's. You're here, we're all in the same boat. And so, you can make fun of each other." Participant 6 explained how the coaches radiate energy, "So, I love the workouts and the coaches are so positive and that, and they create this whole comradery. So, the comradery I think is just as important as the exercise." When asked about how they viewed their fellow boxers, Participant 11 shared, "They're your family, they're fun, fun to see and you joke around and...that makes a big difference in itself. Even without the exercise." Participant 10 shared a more spiritual perspective on how Parkinson's disease impacts the noncontact boxing program, "I felt like there was a similarity, a feeling of family that you would find in a church setting and a feeling of family you would find in people sharing the same fight, which [is] Parkinson's." As participants explained, comradery within the non-contact boxing classes are generated by boxers and coaches alike creating a strong sense of community, family, and unity. 


\section{Framework to Develop Routines}

The occupation of boxing helped participants develop healthy and meaningful patterns of everyday life. This theme is separated into two sub-themes: new routines and well-balanced lifestyle.

New Routines. Non-contact boxing established patterns of behavior that are regular and repetitive to provide structure for daily life. Most participants expressed a desire to maintain a day to day routine initiated by their boxing classes. Participant 8 stated, "I want to take additional classes to kind of get on a routine." Participant 7 shared that non-contact boxing had become a part of their normal routine, stating that it evokes similar feelings as visiting extended family. Routines may be done in solidarity or with others. At Rock Steady Boxing, care partners, also called "cornermen" were welcome to participate in non-boxing classes as long as their loved one does not need assistance to safely complete a workout. Many participants looked forward to establishing routines with their care partners or loved ones. This also offered the opportunity for relationships to be strengthened through the act of sharing a scheduled routine.

Our church offers a 5:30 mass every day. So could do Rock Steady about noon every day and then have an afternoon to do something else and then go to church in the evening. So, we're kind of looking forward as to what our routine might be (Participant 8).

Many participants developed daily or weekly routines with their cornerman. Participant 9 shared their weekly pre-boxing routine. "On Wednesdays, [she] picks me up a little bit early cause my class isn't until $12: 30 \ldots$ So, she shows up little bit early and we stop and 
have lunch somewhere first." Another participant shared what their routine looks like after boxing class.

And we go home, and we get home about 12 or stop for errands on the way home. And walk my dog. Try to walk her twice a day because I want to add more miles in the morning and one at night. And so, we do that when we get home (Participant 16).

The occupation of boxing was new for most of these individuals. A feeling of purpose and desire to participate motivated participants to continue their newly developed routines structured around boxing.

When I know I have something like that to do during a day, I'm more motivated. Okay. You know what I mean? Cause I feel like I have a, there's like a social purpose to it. There's also, um, I know that how good I feel after I exercise and it's something about the group classes that are very motivating to me (Participant 4). Incorporating non-contact boxing into a sequence of occupations and activities throughout the day promoted a satisfying and meaningful routine for many. Participant 2 shared, "I believe I would be way worse condition if I wasn't doing Rock Steady... It's having a place to go to, having a regular schedule, the social part as well and stuff."

Well-balanced Lifestyle. Non-contact boxing classes motivated participants to develop healthy, meaningful routines to live a well-balanced lifestyle.

It's strange, but you talk to a lot of people here and they'll say Parkinson's was this blessing, which is really weird, but I don't know what other kind of thing that could come up in my life that would have made the change, that would have 
gotten me here or would've gotten me somewhere to be able to do all this (Participant 14).

After being diagnosed with Parkinson's disease and starting non-contact boxing, Participant 14 realized the importance of living a well-balanced lifestyle. This participant explained they were an avid runner and had completed half and full marathons. While maybe not as extreme, other participants took extra precautions to ensure they were maintaining a well-rounded and healthy lifestyle. Diet is another factor many participants addressed. Participant 1 stated, "My wife has been very careful about having us on a healthy diet...I am generally pretty strong and able to do stuff. So, I think boxing is part of healthy lifestyle.” Most participants shared that they attended non-contact boxing classes 2-3 times weekly, if not more. Many participants reported a developed exercise routine on days they didn't attend boxing class, whether that be taking time to stretch each morning, go for a walk, or go to the gym. Participant 16 shared, "In a day that I don't come to Rock Steady, I go to Planet Fitness and do the elliptical to stay moving and walk my dog." Participants recognized the benefits that arise when maintaining a healthy routine. Participant 5 shared, "the biggest impact is just overall physical strength and health and wellbeing, you know." Participant 4 shared how having a healthy physical routine had an overall positive impact on their life. Participant 4 stated, "It makes me more of like a happy person in general.” Promoting and maintaining a well-balanced lifestyle enhances participation in many occupations boosting well-being, happiness, and quality of life. 


\section{Discussion and Conclusion}

\section{Discussion}

This qualitative capstone project described the perceptions of non-contact boxing on daily life and occupational participation at home among individuals with Parkinson's disease. This research topic aimed to answer two questions: 1) how non-contact boxing impacts daily life, and 2) how non-contact boxing impacts occupational participation at home. Exploring participants' $(n=17)$ responses demonstrated the individual nature of each person's experience with both Parkinson's disease and non-contact boxing. The first part of the research question, how non-contact boxing impacts daily life, provided consistent results gathered into the three themes. However, the second part of the research question, how non-contact boxing impacts occupational participation at home, did not produce consistent results, according to the participants $(n=17)$ recruited from the Rock Steady Boxing Headquarters gym. The following discussion will dive deeper into both parts of the research topic.

\section{1) How Non-contact Boxing Impacts Daily Life}

The three themes are consistent across the entire data set (i.e., all 17 participants). There were no significant differences found between the four levels of classes. Therefore, according to the researcher, the participants in the PD1 classes did not provide responses different enough to recognize a change in pattern when compared to participants in the PD3 classes. The main difference the researcher noticed when conducting interviews, was that participants in higher level classes (i.e., PD1 \& PD2) were able to sustain a higher level of attention and provided more appropriate responses related to the question compared to participants in lower level classes (i.e., PD3 \& PD4). Rosińczuk \& 
Kołtuniuk (2017) address cognitive impairments (e.g., dementia and language difficulties) that have a significant impact on how individuals with Parkinson's disease view their quality of life. Recent literature explores how common deficits in language among those with Parkinson's disease impact important occupations such as social engagements, including participation in interviews (Wolff \& Benge, 2019).

The first two themes, "Psychological benefits," and "Sense of community" relate to aspects of psychosocial health. Psychosocial health encompasses emotional, spiritual, mental, and social dimensions of health and well-being. As referenced in the literature review, Sheehy and colleagues (2017) explored the effects of boxing on social supports and challenges among individuals with Parkinson's disease. They concluded that participation in a group boxing program provides relief from anxiety and depression and strengthens feelings of inspiration and support (Sheehy et al., 2017). The results from this study are with results of this capstone project. However, this capstone identified patterns of how non-contact boxing installs confidence, positivity, and energy to tackle challenges individuals with Parkinson's disease face according to the participants $(n=17)$ of this study. The literature addresses that physical rehabilitation fosters social bonding and promotes social participation (Lizon et al., 2017). This capstone project strengthens research on this topic while addressing how non-contact boxing provides a gateway to new social relationships decreasing loneliness and isolation in the home. According to the participants in this study, the atmosphere of community and family expands beyond the physical boundaries of the non-contact boxing gym.

It is important to address the theme "Framework to Develop Routines" includes the subtheme "New Routines". The researcher purposefully chose this title rather than 
simply "Routines" as 14 out of 17 participants are retired. Employment provides structure and routine to a day without much thought (e.g., wake up, get ready for the day, go to work, come home, eat, relax, go to bed). When this daily routine is suddenly stopped, individuals look to fill their time with other activities. Although not explicitly stated, the researcher understood that many participants use non-contact boxing classes to provide purposeful structure to their newly developed routines. The literature explores the benefits of routine on the community-dwelling older adults with cognitive impairments due to neurodegenerative conditions. Routines increase the efficiency of daily occupations due to the minimal stress on cognitive processes, such as working memory and decision making (Sanders \& Van Oss, 2013). Important occupations within a daily routine, such as taking medication, become a more automated response when embedded into an everyday routine (Sanders \& Van Oss, 2013).

\section{2) How Non-contact Boxing Impacts Occupational Participation at Home}

When the researcher asked participants to elaborate on how non-contact boxing has impacted specific occupations at home, the original research question, many participants had trouble pinpointing specifics. Some participants stated it was hard to know what symptoms where secondary to Parkinson's disease and what symptoms to constitute to old age or another underlying condition. For example, when the researcher asked questions regarding self-care routines or the ability to complete household tasks and how these activities have been affected by both Parkinson's disease and boxing, participants would state that they were not sure. They would address challenges they may face when participating in these occupations, however, many times they were not able to provide reasoning and fully answer the researcher's question. For example, they would 
say something like, "is that Parkinson's or is it a good ole cold?", or "sometimes in my mind I get the two confused (talking about Parkinson's disease and another underlying condition)," or “what's a physical element versus Parkinson's?” or “what symptom goes with what disease?" or "that's unrelated to Parkinson's," or "it might be related to age," or "it's very inconsistent." Participants had trouble pinpointing answers to the interviewer's questions. The nature of the disease does encompass fluctuations in symptoms, moods, and mental functions (Son \& Choi, 2018). Mental function includes attention, memory, and perceptions. It is important to note that the interviews in this study may have been completed on what the participant would call a "good day" or when a participant was having an "off day." The information in this research study is purely data derived from 17 different individuals interviewed on a pre-scheduled date.

Medication is another factor to address that made it difficult for participants to answer how boxing impacted their home occupations. Most participants reported taking Parkinson's related medications. This too suggests challenges in participants' abilities to distinguish what benefits are due to participation in non-contact boxing classes as opposed to their medications. The subjective outcome measure of interview and gathering participants' perceptions rather than taking objective measurements/observations gathered in this study will be addressed further in the limitations section of the paper.

Although overall themes were identified on non-contact boxings impact on overall health and wellness, discussion of the direct impact of non-contact boxing on specific occupations in the home environment did not conclude consistent results. Due to the nature of a qualitative study, the student researcher's topic addresses participants' perceptions rather than objective measurements. The researcher did not answer medical 
questions and simply acknowledged struggles many participants addressed. This challenge leads to the question that perhaps participants do not fully understand how Parkinson's disease has impacted their ability to participate in ADLs and IADLs in the home environment. Or perhaps they do not feel comfortable speaking on behalf of physical challenges secondary to Parkinson's disease due to unknown reasons. These may be simply assumptions, but reasons why the researcher faced challenges when developing themes related to self-care, home maintenance, and other household tasks and exploring alternative methods to gather this information may provide a focus for future research to explore.

\section{Implications for Occupational Therapy}

The PEOP model that was introduced in the introduction section of this paper built the framework for developing this research project. The PEOP model was used as a template to address occupational performance and participation concerns of individuals with Parkinson's disease and to discuss both intrinsic and extrinsic factors relating to performance. Intrinsic factors include physiological, cognitive, spiritual, neurobehavioral, and psychological elements; extrinsic factors include social support, culture and values, built environment and technology, and the natural environment (Smith \& Hudson, 2012). After completion of the interviews, it is fair to say that all of these intrinsic and extrinsic factors came up at least once during interviews. Three factors (i.e., psychological, social support, and culture and values) directly relate to the three overarching themes: psychological benefits, sense of community, and framework to develop routines. This confirms the researcher's notion to use the PEOP model to guide 
this research project and may be used as a suggestion for occupational therapists to utilize this model when working with individuals within this population.

The Model of Human Occupation (MOHO) is an occupation-focused, theorydriven, client-centered approach to occupational therapy practice (Forsyth et al., 2014). This model understands occupational therapy as a process in which practitioners support client participation in occupations shaped by 1) the motivation for occupation, 2) habituation, 3) performance capacity, and 4) social and physical environment (Forsyth et al., 2014). MOHO provides the framework to apply results of this study to clinical practice (Forsyth et al., 2014). Occupational therapists can use this model to identify a client's strengths and limitations, plan, and implement a course of action to further promote participation in meaningful occupations despite challenges individuals with Parkinson's disease might face. Personal causation is a term defined by MOHO and relates to how an individual feels about themselves (Forsyth et al., 2014). The first two themes identified in this study, psychological benefits and sense of community, enhances an individual's personal causation and motivation to participate in occupations. The third theme identified in this study, framework to develop routines, relates to MOHO's idea of habituation, referring to the process of organizing patterns and routines (Forsyth et al., 2014). Non-contact boxing provides the framework to develop routines, and occupational therapists may help individuals further establish patterns of living to inspire individuals to live a fulfilling life.

\section{Limitations and Future Considerations}

The researcher initially limited the scope of this study to the impact of noncontact boxing on participants' everyday life and occupational participation in the home 
environment. The researcher aimed to maintain an occupation focus and explore how one occupation (i.e., non-contact boxing) impacted their performance and participation in other occupations (i.e., ADLs, IADLs, sleep, intimacy, leisure). Questions were limited to those exclusively involving the home environment to control the volume of data. However, if the research topic and therefore interview questions were broader and allowed for the impact of the non-contact boxing in general, more themes and therefore further areas of future research may have emerged. Subsequent research exploring experiences of how non-contact boxing impacts everyday life from individuals across states and internationally would support transferability of the results to a wider population (Portney \& Watkins, 2009).

There are a variety of techniques to increase credibility and consistency of qualitative data (Portney \& Watkins, 2009). Member checking was not utilized, although implementation of this strategy would have added credibility to the research (Portney \& Watkins, 2009). Although the researcher was knowledgeable on these techniques, capstone project time constraints did not allow for these strategies to be implemented. The student researcher also wanted to respect the limited time and energy of the participants. Interviews were the only method of data collection used in this study. Interviews provide a strong understanding of participant's perspectives but fail to gather information other than what the interviewer shares (Portney \& Watkins, 2009). Triangulation strengthens research by providing a second source of data, such as direct observation (Portney \& Watkins, 2009). It is recommended that future research adds in extended observations in the home to strengthen the interpretation of data providing more objective measurements. 
Quantitative research would also help to further understanding of how noncontact boxing impacts individuals with Parkinson's disease, as research drawn from personal experiences alone is lacking objective data. Certified occupational therapists have the ability to perform assessment measures within the home of individuals with Parkinson's disease to develop a more factual understanding of participant's ability to participate in daily occupations.

During interviews, several participants brought up their responsibility to care for either their parent(s) and/or their dependent child(ren). Early onset Parkinson's disease increases this likelihood. Individuals explore ways to care for dependents along with navigating their own disease. Future research could focus on the topic of how individuals with Parkinson's, or any neurodegenerative condition, manage the balance of care. Secondly, caregivers of those with Parkinson's disease, such as spouses, children, etc. could provide valuable information on their loved one's ability to participate in everyday occupations and changes in routines as they are typically right there with them. Future research exploring caregivers' perspectives would further support this topic of research.

\section{Dissemination}

The student researcher disseminated in April by presenting a PowerPoint and video presentation to the staff, coaches, and participants of Rock Steady Boxing. This presentation and a flyer were shared virtually by Rock Steady Boxing Headquarters gym staff to the Headquarters gym social media page and e-mail list. The PowerPoint and video presentation included information on the capstone project and experience, methods and results of the research, how the study relates to occupational therapy, and words of encouragement to Rock Steady Boxers. The flyer and PowerPoint presentation can be 
found in Appendix $\mathrm{G}$ and $\mathrm{H}$, respectively. A copy of the poster presented for fulfillment of the occupational therapy doctorate degree was shared with Rock Steady Boxing for them to display at their Headquarters gym in Indianapolis, IN. See Appendix I for the poster. The student researcher will submit this capstone project to be considered for journal publication in efforts to add this capstone project to the current literature. See Appendix $\mathrm{J}$ for reasoning for journal publication.

\section{Conclusions}

This capstone project contributes to the literature of Parkinson's disease through exploring the impact of non-contact boxing on everyday life and occupational participation among those with Parkinson's disease. This project adds to the literature by shifting the perspective to view participants' thoughts, allowing occupational therapists and other health professionals the opportunity to better serve individuals with Parkinson's disease to promote engagement in meaningful occupations and increase quality of life. Further research is necessary to continue learning more about the impact of non-contact boxing on occupational participation among those with Parkinson's disease.

\section{Acknowledgements}

Special thanks to the coaches and staff at the Rock Steady Boxing HQ gym and all of the boxers who contributed to my capstone experience in so many ways. I also want to thank my mentor Christine Childers, PT, BSc (Hons), MS, PhD for providing me with continued support and guidance. 


\section{References}

Amara, A. W., \& Memon, A. A. (2018). Effects of exercise on non-motor symptoms in Parkinson's disease. Clinical Therapeutics, 40(1), 8-15. https://doi.org/10.1016/j.clinthera.2017.11.004

American Occupational Therapy Association. (2014). Occupational therapy practice framework: Domain and process (3rd ed). American Journal of Occupational Therapy, 68(1), S1-S48. https://doi.org/10.5014/ajot.2014.682006

Braun, V. \& Clarke, V. (2006) Using thematic analysis in psychology. Qualitative Research in Psychology, 3(2), 77-101. http://dx.doi.org/10.1191/1478088706qp063oa

Choi, Y.-I., Song, C.-S., \& Chun, B.-Y. (2017). Activities of daily living and manual hand dexterity in persons with idiopathic Parkinson's disease. Journal of Physical Therapy Science, 29(3), 457-460. https://doi.org/10.1589/jpts.29.457

Combs, S. A., Diehl, M. D., Chrzastowski, C., Didrick, N., McCoin, B., Mox, N., Staple, W. H., \& Wayman, J. (2013). Community-based group exercise for persons with Parkinson's disease: A randomized controlled trial. NeuroRehabilitation, 32(1), 117-124. https://doi.org/10.3233/NRE-130828

Combs, S. A., Diehl, M. D., Staples, W. H., Conn, L., Davis, K., Lewis, N., \& Schaneman, K. (2011). Boxing training for patients with Parkinson's disease: A case series. Physical Therapy, 91(1), 132-142. https://doi.org/10.2522/ptj.20100142

"Dedoose Version 8.3.17 web application for managing, analyzing, and presenting qualitative and mixed method research data" (2020). Los Angeles, CA: 
SocioCultural Research Consultants, LLC. https://app.dedoose.com/App/?Version=8.3.17

Ebersbach, G., Ebersbach, A., Edler, D., Kaufhold, O., Kusch, M., Kupsch, A., \& Wissel, J. (2010). Comparing exercise in Parkinson's disease-the Berlin BIG study. Movement Disorders, 25(12), 1902-1908. https://doi.org/10.1002/mds.23212

Feng, X., \& Behar-Horenstein, L. (2019). Maximizing NVivo utilities to analyze openended responses. The Qualitative Report, 24(3), 563-571. Retrieved from https://nsuworks.nova.edu/tqr/vol24/iss3/10

Fleisher, J., Barbosa, W., Sweeney, M. M., Oyler, S. E., Lemen, A. C., Fazl, A., Ko, M., Meisel, T., Friede, N., Dacpano, G., Gilbert, R. M., Di Rocco, A., \& Chodosh, J. (2018). Interdisciplinary home visits for individuals with advanced Parkinson's disease and related disorders: Home visits for people with Parkinson's disease. Journal of the American Geriatrics Society, 66(6), 1226-1232. https://doi.org/10.1111/jgs.15337

Forsyth, K., Taylor, R. R., Kramer, J. M., Prior, S., Richie, L., Whitehead, J. Owen, C., \& Melton, J. (2014). The Model of Human Occupation. In B. A. Boyt Schell, G. Gillen, M. E. Scaffa, \& E. S. Cohn (Eds.), Willard \& Spackman's Occupational Therapy (12 $2^{\text {th }}$ ed., pp. 505-526). Lippincott Williams \& Wilkins.

Foster, E. R., Bedekar, M., \& Tickle-Degnen, L. (2014). Systematic review of the effectiveness of occupational therapy-related interventions for people with Parkinson's disease. American Journal of Occupational Therapy, 68(1), 39-49. https://doi.org/10.5014/ajot.2014.008706 
Foster, E. R. (2014). Instrumental activities of daily living performance among people with Parkinson's disease without dementia. American Journal of Occupational Therapy, 68(3), 353. https://doi.org/10.5014/ajot.2014.010330

Fox, C., Ebersbach, G., Ramig, L., \& Sapir, S. (2012). LSVT LOUD and LSVT BIG: Behavioral treatment programs for speech and body movement in Parkinson's disease. Parkinson's Disease, 2012, 1-12. https://doi.org/10.1155/2012/391946

Kegelmeyer, D., Ellis, T., Esposito, A., Gallagher, R., Harro, C. C., Hoder, J., Hussey, E., \& O’Neal, S. (2015). Measurement characteristics and clinical utility of the Parkinson's disease quality of life measure (39- and 8-item versions) in individuals with Parkinson disease. Archives of Physical Medicine and Rehabilitation, 96(8), 1551-1552. https://doi.org/10.1016/j.apmr.2015.02.022

Kwok, J. Y. Y., Kwan, J. C. Y., Auyeung, M., Mok, V. C. T., \& Chan, H. Y. L. (2017). The effects of yoga versus stretching and resistance training exercises on psychological distress for people with mild-to-moderate Parkinson's disease: Study protocol for a randomized controlled trial. Trials, 18(1). https://doi.org/10.1186/s13063-017-2223-X

Laliberte, R. (2017). Parkinson's on the ropes: Exercise programs incorporating boxing skills may help manage symptoms of Parkinson's disease. Here's how they work. Neurology Now, 39-41. http://www.neurologynow.com

Lizon, K., Cholewa, J., Uher, I. (2017). Influence of physical rehabilitation on social networks and quality of life among people with Parkinson's disease. Journal of Physical Education \& Health, 6(10), 35-42.

LSVT Global (2020). What is LSVT BIG? https://www.lsvtglobal.com/LSVTBig 
Morberg, M. B., Jensen, J., Bode, M., \& Wermuth, L. (2014). The impact of high intensity physical training on motor and non-motor symptoms in patients with Parkinson's disease (PIP): A preliminary study. NeuroRehabilitation, 35(2), 291298. https://doi.org/10.3233/NRE-141119

Miertová, M., Tomagová, M., Jarošová, M., \& Kiabová, L. (2014). Self-care in patients with Parkinson's disease. Central European Journal of Nursing and Midwifery, 5 (2), 54-62.

Parkinson's Foundation (2020). Stages of Parkinson's. https://www.parkinson.org/Understanding-Parkinsons/What-is-Parkinsons/Stages$\underline{\text { of-Parkinsons }}$

Perry, S. I. B., Nelissen, P. M., Siemonsma, P., \& Lucas, C. (2019). The effect of functional-task training on activities of daily living for people with Parkinson`s disease, a systematic review with meta-analysis. Complementary Therapies in Medicine, 42, 312-321. https://doi.org/10.1016/j.ctim.2018.12.008

Portney, L. G., \& Watkins, M. P. (2009). Foundations of clinical research: Applications to practice $\left(3^{\text {rd }}\right.$ ed.). Pearson Education.

Rosińczuk, J., \& Kołtuniuk, A. (2017). The influence of depression, level of functioning in everyday life, and illness acceptance on quality of life in patients with Parkinson's disease: A preliminary study. Neuropsychiatric Disease and Treatment, 13, 881-887. https://doi.org/10.2147/NDT.S132757

Sanders, M. J., \& Van Oss, T. (2013). Using daily routines to promote medication adherence in older adults. American Journal of Occupational Therapy, 67, 91-99. http://dx.doi.org/10.5014/ajot.2013.005033 
Serrano-Dueñas, M., Bravo, R., Merchán, T., \& Serrano, M. (2018). Fatigue in Parkinson's disease: Metric properties of the fatigue impact scale for daily use (DFIS), and its impact on quality of life. Clinical Neurology and Neurosurgery; Assen, 169, 12-15. http://dx.doi.org.prxusa.lirn.net/10.1016/j.clineuro.2018.03.020

Shah, S., \& Nolen, A. (2006). Movement deficits in Parkinson's disease and restorative occupational therapy. New Zealand Journal of Occupational Therapy, 53(2), 1219.

Sheehy, T. L., McDonough, M. H., \& Zauber, S. E. (2017). Social comparisons, social support, and self-perceptions in group exercise for people with Parkinson's disease. Journal of Applied Sport Psychology, 29(3), 285-303. https://doi.org/10.1080/10413200.2016.1266711

Shulman, L. M., Katzel, L. I., Ivey, F. M., Sorkin, J. D., Favors, K., Anderson, K. E., Smith, B. A., Reich, S. G., Weiner, W. J., \& Macko, R. F. (2013). Randomized clinical trial of 3 types of physical exercise for patients with Parkinson disease. Journal of American Medical Association Neurology, 70(2), 183-190. https://doi:10.1001/jamaneurol.2013.646

Sidaway, B., Ala, B., Baughman, K., Glidden, J., Cowie, S., Peabody, A., Roundy, D., Spaulding, J., Stephens, R., \& Wright, D. L. (2016). Contextual interference can facilitate motor learning in older adults and in individuals with Parkinson's disease. Journal of Motor Behavior, 48(6), 509-518. https://doi.org/10.1080/00222895.2016.1152221 
Smith, D., \& Hudson, S. (2012). Using the person-environment-occupational performance conceptual model as an analyzing framework for health literacy. Journal of Communication in Healthcare, 5(1), 11-13. https://doi.org/10.1179/1753807611Y.0000000021

Son, H. G., \& Choi, E.-O. (2018). The effects of mindfulness meditation-based complex exercise program on motor and nonmotor symptoms and quality of life in patients with Parkinson's disease. Asian Nursing Research, 12(2), 145-153. https://doi.org/10.1016/j.anr.2018.06.001

Sperens, M., Hamberg, K., \& Hariz, G.-M. (2018). Challenges and strategies among women and men with Parkinson's disease: Striving toward joie de vivre in daily life. British Journal of Occupational Therapy, 81(12), 700-708. https://doi.org/10.1177/0308022618770142

Sturkenboom, I. H. W. M., Graff, M. J. L., Hendriks, J. C. M., Veenhuizen, Y., Munneke, M., Bloem, B. R., \& der Sanden, M. W. N. (2014). Efficacy of occupational therapy for patients with Parkinson's disease: A randomised controlled trial. The Lancet Neurology, 13(6), 557-566. https://doi.org/10.1016/S1474-4422(14)70055-9

Suchowersky O, Gronseth G, Perlmutter J, Reich S, Zesiewicz T, \& Weiner WJ. (2006). Practice parameter: Neuroprotective strategies and alternative therapies for Parkinson's disease (an evidence-based review): Report of the Quality Standards Subcommittee of the American Academy of Neurology. Neurology, 66(7), 976982. https://doi.org/10.1212/01.wnl.0000206363.57955.1b

“Temi” (2020). https://www.temi.com 
Toygar, I., Yildirim, Y., Ozer, S., \& Ismail, T. (2018). Dance therapy in the rehabilitation of the Parkinson's disease. International Journal of Caring Sciences, 11(3), 20052008.

Uhrbrand, A., Stenager, E., Pedersen, M. S., \& Dalgas, U. (2015). Parkinson's disease and intensive exercise therapy - a systematic review and meta-analysis of randomized controlled trials. Journal of the Neurological Sciences, 353(1-2), 919. https://doi.org/10.1016/j.jns.2015.04.004

University of St. Augustine for Health Sciences (2020). Institutional Review Board. https://my.usa.edu/ICS/Academics_2017-08-22T17-14-45-332/IRB/

Vaartio-Rajalin, H., Rauhala, A., \& Fagerström, L. (2019). Person-centered home-based rehabilitation for persons with Parkinson's disease: A scoping review. International Journal of Nursing Studies, 99. https://doi.org/10.1016/j.ijnurstu.2019.103395

Wolff, L., \& Benge, J. (2019). Everyday language difficulties in Parkinson's disease: Caregiver description and relationship with cognition, activities of daily living, and motor disability. American Journal of Speech-Language Pathology, 28(1), 165-173. https://doi.org/10.1044/2018_AJSLP-18-0091 


\author{
Appendix A \\ IRB Exempt Approval Letter
}

\title{
UNIVERSITY OF ST. AUGUSTINE \\ F O R H H E A L T H S C I E N C E S
}

To:

Dr. Susan MacDermott Principal Investigator

Kristen Bignal Student Investigator

From: Iman Akef Khowailed, DSc.

Date: $\quad$ November $8^{\text {th }}, 2019$ - Exempt Review

Re: $\quad$ IRB EXEMPT Protocol Application

Your IRB application submitted titled, "Perceived Impact of Non-Contact Boxing Program on Daily Life and Occupational Participation at Home Among Individuals with Parkinson's Disease” falls under the Exempt Review category as listed in 45 CFR §46.

- $\quad$ This application was reviewed as exempt as written on November $8^{\text {th }}, 2019$.

○ Exempt Category 45 CFR $\$ 46.104$

- The protocol number is 1105-019.

Please note that any changes to the exempt protocol must be re-reviewed by the IRB committee.

As Chair of the California IRB, I have reviewed this protocol and determined that it qualifies as exempt per 45 CFR $\S 46.104$.

Iman Akef Khowailed $\begin{aligned} & \text { Digitally signed by Iman Akef Khowailed } \\ & \text { DN: cn=Iman Akef Khowailed, o, ou, } \\ & \text { email=iakef@usa.edu, c=US }\end{aligned}$

Iman Akef Khowailed, DSc

California IRB Chair

Cell: (310) 666-0108

Email: iakef@usa.edu

PLEASE NOTE: It is the responsibility of the primary researcher to amend their IRB protocol to add any future individuals who will be handling research data before they join the study. These individuals must be added to the protocol via an approved protocol amendment and must complete the required CITI online course before they may handle research data or interact with human research subjects. If new individuals are not added to the protocol and trained PRIOR TO ANY HUMAN SUBJECTS CONTACT, the IRB may revoke the primary researcher's approval to conduct the research project.

*Note: Please keep a copy of this Approval with your protocol documents and retain per the USAHS records retention schedule.

A copy of all approvals must be kept in the Research Facility or office to be viewed during inspections. 


\section{Appendix B \\ Recruitment Script}

Hello everyone and thank you for welcoming me to participate in your boxing class today. My name is Kristen and I am an occupational therapy student. I am completing a research study for my doctorate project on the topic of how non-contact boxing classes impact your daily life at home. I am looking for people to sign up for a one-hour time slot to participate in an interview with me and complete a questionnaire. I will ask you questions about your experience with this program and how it has impacted your everyday life at home. If you are interested in signing up, there are flyers at the front desk where you can learn more information and my contact information. Your interest will remain confidential. I'll stick around, if you have any questions about my project feel free to ask. Thank you! 


\section{Appendix C}

Recruitment Flyer

Hello! My name is Kristen and I am an occupational therapy student. I am completing my doctorate project on the topic of the perceived impact of non-contact boxing on daily life and occupational participation at home among individuals with Parkinson's disease. Occupational participation is any form of activity that you do that occupies your time. This includes getting ready in the morning, cooking dinner, enjoying leisure interests, and even going to sleep. I hope to bring awareness to the importance of the ability to participate in meaningful activities.

I am looking for people to volunteer for an interview with me and to complete a questionnaire. It will take a total of one hour of your time. I will ask you questions about your experience with this boxing program and how it has impacted your everyday life at home.

If you are interested in participating in the study or would like more information, please email, call, or come talk to me. Your interest will remain confidential.

Thank you,

Kristen Bignal

317-460-4773

k.bignal@usa.edu 


\section{Appendix D \\ Consent Form}

\section{$\underline{\text { IRB }}$}

University of St. Augustine for Health Sciences

Out of town: 1-866-577-3731 x2468; Local: 1-760-591-3012 x2468

IRB Informed Consent Form, IRB \#

\section{CONSENT FOR RESEARCH PARTICIPATION}

Title of Project: Perceived impact of non-contact boxing on daily life and occupational participation at home among individuals with Parkinson's disease.

IRB Number:

Student Principal Investigator:

Kristen Bignal, OTDS

317-460-4773

k.bignal@usa.edu

5343 Carrollton Ave Indianapolis, IN 46220

Supervising Principal Investigator for Student:

Susan MacDermott, OTD, OTR/L

760-591-3012 x 2404

smacdermott@usa.edu

700 Windy Point Dr, San Marcos, CA 92069

You are being asked to participate in a research study. The box below highlights key information about this research for you to consider when making a decision whether or not to participate. Carefully consider this information and the more detailed information provided below the box. Please ask questions about any of the information you do not understand before you decide whether to participate.

\section{Key Information for You to Consider}

- Voluntary Consent. You are being asked to volunteer for a research study. It is up to you whether you choose to participate or not. There will be no penalty or loss of benefits to which you are otherwise entitled if you choose not to participate or discontinue participation.

- Purpose. The purpose of this research is to determine the perception of individuals with Parkinson's disease of how non-contact boxing impacts their daily life and participation in meaningful activities at home.

- Duration. It is expected that your participation will last about one hour.

- Procedures and Activities. You will be asked to participate in an interview with the student researcher and complete a questionnaire.

- Risks. There are no foreseeable risks or discomforts to participate.

- Benefits. There are no benefits that may be expected of your participation. 
- Alternatives. Your participation is voluntary, and participants can determine whether they want to complete the interview and/or the questionnaire, or not participate.

\section{Why is this research being done?}

As a student in an occupational therapy program, I am completing my doctorate project on the topic of the perceived impact of non-contact boxing on daily life and occupational participation at home among individuals with Parkinson's disease. Occupational participation is any form of activity that you do that occupies your time. This includes getting ready in the morning, cooking dinner, enjoying leisure interests, and even going to sleep. I hope to bring awareness to the importance of the ability to participate in meaningful activities.

\section{What Will Happen in This Research Study}

The study will consist of an interview and a questionnaire. One hour of your time is needed to participate in this study to complete:

- The Parkinson's Disease Questionnaire (PDQ-39) 15 minutes

- Interview/conversation with the student researcher $\sim 45$ minutes

The ways we will protect your privacy and confidentiality are described in a separate section later in this form.

I will make an audio recording so I can go back to listen to this interview at a later date. By agreeing to participate, you are consenting to having this interview audio taped.

\section{Costs}

There are no costs to you for being in this research study.

\section{Payments}

You will not be paid for being in this study.

\section{$\underline{\text { Confidentiality }}$}

We will not record your name or any information that shows your identity. You will not be signing this form.

We will store your information in ways we think are secure. We will store electronic files in computer systems with password protection. However, we cannot guarantee complete confidentiality.

\section{Subject's Rights}

By consenting to be in this study you do not waive any of your legal rights. Consenting means that you have been given information about this study and that you agree to participate in the study. You will be given a copy of this form to keep. 
If you do not agree to be in this study or if at any time you withdraw from this study you will not suffer any penalty or lose any benefits to which you are entitled. Your participation is completely up to you. Your decision will not affect your ability to get health care or payment for your health care. It will not affect your enrollment in any health plan or benefits you can get.

\section{Questions}

The investigator or a member of the research team will try to answer all of your questions. If you have questions or concerns at any time, contact Kristen Bignal at 317-460-4773.

You may also call 737-202-3343 or email eardolino@usa.edu. You will be talking to Elizabeth Ardolino, the chairperson of the IRB at the University of St. Augustine for Health Sciences. The IRB is a group that helps monitor research. You should call or email the IRB if you want to find out about your rights as a research subject. You should also call or email if you want to talk to someone who is not part of the study about your questions, concerns, or problems.

By agreeing to be in this research, you are indicating that you have read this form (or it has been read to you), that your questions have been answered to your satisfaction, and that you voluntarily agree to participate in this research study.

THIS PROJECT HAS BEEN REVIEWED AND APPROVED BY THE UNIVERSITY OF ST. AUGUSTINE FOR HEALTH SCIENCES INSTITUTIONAL REVIEW BOARD FOR THE PROTECTION OF HUMAN SUBJECTS.

IF YOU HAVE QUESTIONS OR CONCERNS, THOSE QUESTIONS OR CONCERNS SHOULD BE DIRECTED TO THE INSTITUTIONAL IRB CHAIR, DR. ELIZABETH ARDOLINO, EMAIL: EARDOLINO@USA.EDU, PHONE: 737-202-3343. 


\section{Appendix E Semi-Structured Interview}

\section{Introduction Script:}

Hello, my name is Kristen and I am an occupational therapy student. This interview is part of my research study. Thank you for participating and being here today. Your participation is voluntary, and you may withdraw from the study at any time. Occupational therapy helps people do the things they want and need to do on a daily basis regardless of abilities or illnesses. This includes getting ready in the morning, cooking a meal and participating in mealtime with family and friends, hobbies, and anything else that you like to do.

I am studying how fighters, like you, believe this non-contact boxing program impacts your daily life at home and participation in meaningful activities. I have some questions to guide our discussion and I would like you to answer to the best of your abilities. At any time, please let me know if you have any questions. There is an audio recorder that will record our interview. This will help me so I can go back to hear what you said. Do you have any questions before we begin?

\section{Interview Questions:}

1. How long has it been since you were diagnosed with Parkinson's disease?

2. How long have you been participating in the program?

3. Share with me your experience with the boxing program.

4. Can you please describe your current self-care routine? These are the activities you do to take care of yourself including going to the bathroom, showering, dressing, grooming, and hygiene.

5. Did your self-care routine change as a result of your Parkinson's disease diagnosis?

6. Has your self-care routine been further influenced/changed since participating in the boxing program?

7. Can you please describe any household tasks you normally complete? This includes cleaning, cooking, taking out the trash, etc.

8. Did your ability to complete any household tasks change as a result of your Parkinson's disease diagnosis?

9. Has your ability to complete any household tasks been further influenced/changed since participating in the boxing program?

10. Did your ability to sleep change as a result of your Parkinson's disease diagnosis?

11. Have your sleep patterns changed since participating in the boxing program?

12. Did your ability to be intimate change as a result of your Parkinson's disease diagnosis?

13. Has your ability to be intimate changed since participating in the boxing program?

14. Please describe your hobbies and leisure interests.

15. Were there hobbies that you were no longer able to participate in after your diagnosis of Parkinson's disease?

16. Since participating in the boxing program, are there hobbies you can now do again or any new hobbies you have started?

17. Do you have anything else you would like me to know about how this boxing program has impacted your day to day life? 
Appendix F

Grant Approval Letter

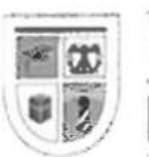

UNIVERSIT

January 22,2020

Re: OTD Capstone Grant

Dear Kristen Bignal,

The University of St Augustine for Health Sciences has approved your grant request for the full amount of $\$ 200$. We are pleased to be able to support you in your continued scholarship efforts as you complete your Doctor of Occupational Therapy Capstone. Instructions will be provided via email by the program assistant to complete financial paperwork. Please save and send receipts to your campus Doctoral Coordinator, Susan MacDermott, when items are purchased.

Thank you for your efforts and we look forward to hearing about your progress.

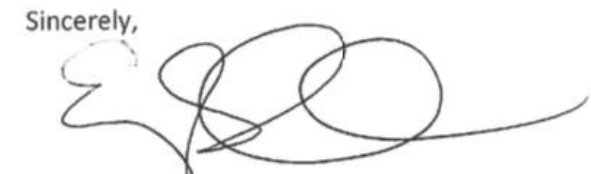

Erin Schwier EdD, OTD, OTR/L

Program Director, Associate Professor

Occupational Therapy Programs, CA Campus

University of St. Augustine for Health Sciences 


\section{Appendix G}

Flyer

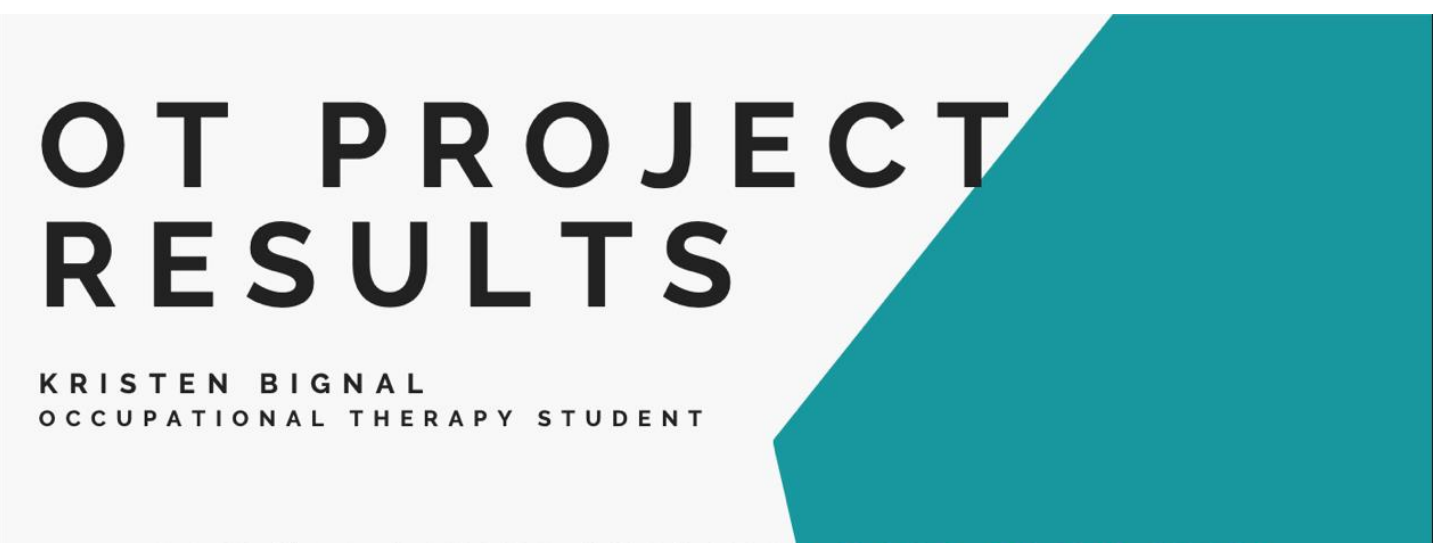

HOW HAS BOXING IMPACTED YOUR DAILY LIFE?

happyc/shelp friendship motivated

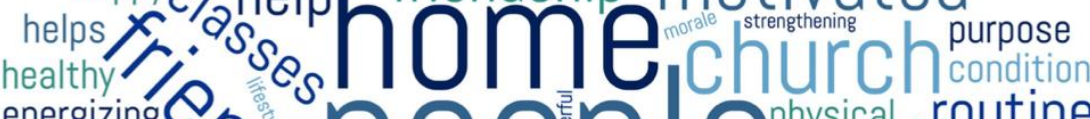
energizing

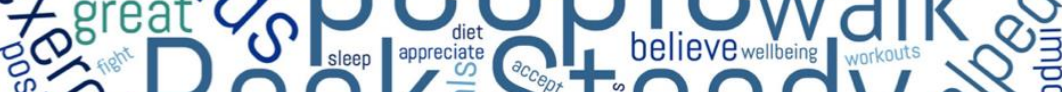

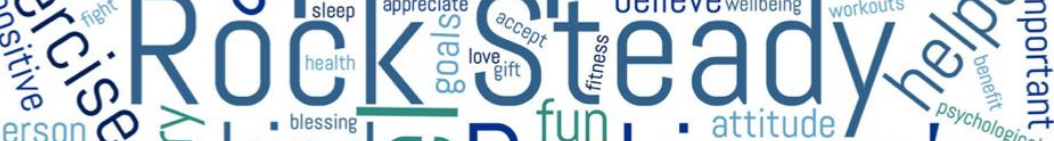
person $\mathrm{C} \frac{\mathrm{C}}{\mathrm{C}}$ / blessing simiarity

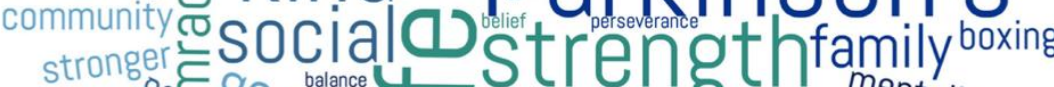
stronger \&

\section{THANK YOU}

THANKS TO EVERYONE AT RSB HQ GYM WATCH THE VIDEO! AND LET ME KNOW IF YOU HAVE ANY QUESTIONS K.BIGNAL@USA.EDU 
Appendix H

PowerPoint Presentation

$4 / 16 / 20$

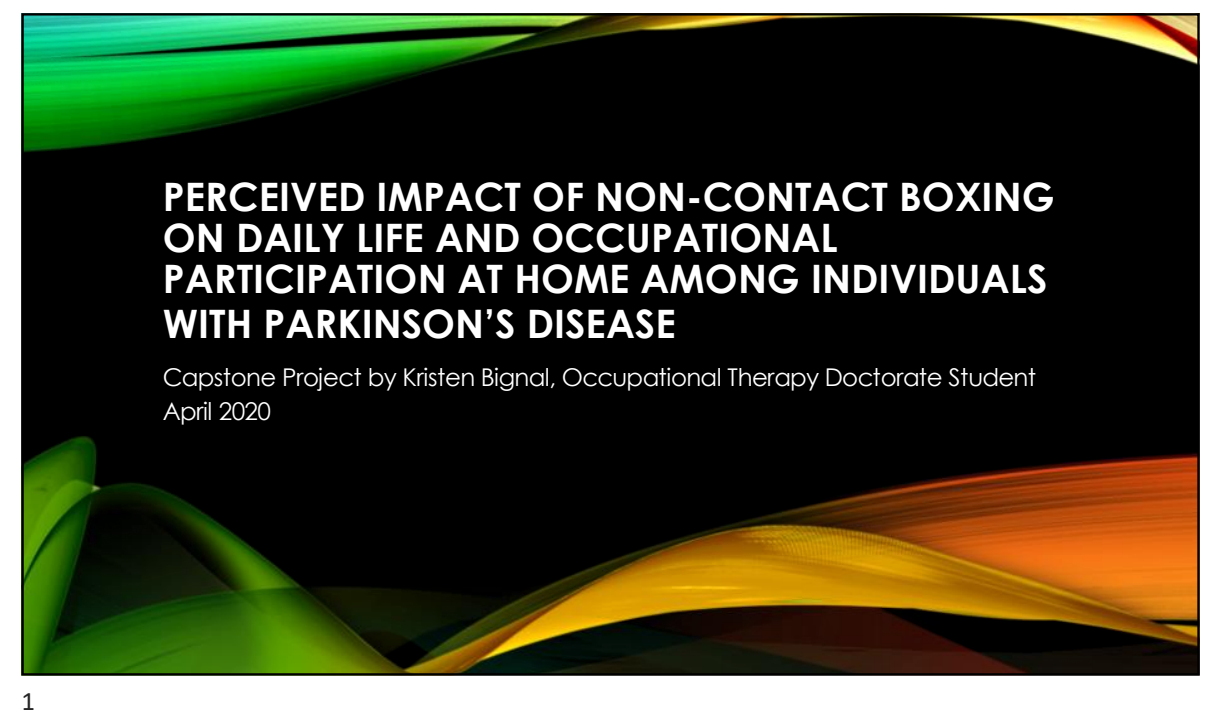

IRB APPROVED

- $\mathrm{IRB}=$ Institutional Review Board

- Participant Confidentiality 


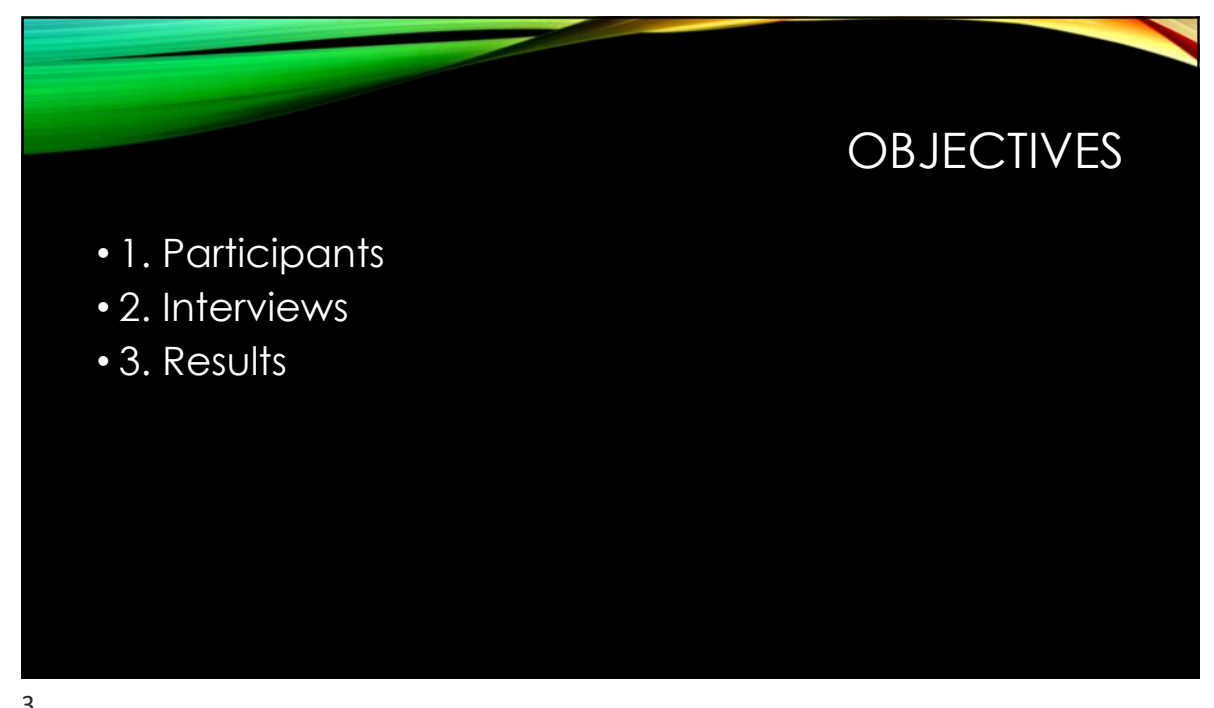

3

\section{PARTICIPANTS}

- 17 participants

- 6 PD1

- 5 PD2

- 5 PD3

- 1 PD4

- Over 40 year age gap between youngest and oldest

- 14 male, 3 female

- Diagnosed 2004-2019

- Rock Steady Boxing member since 2007-2019 


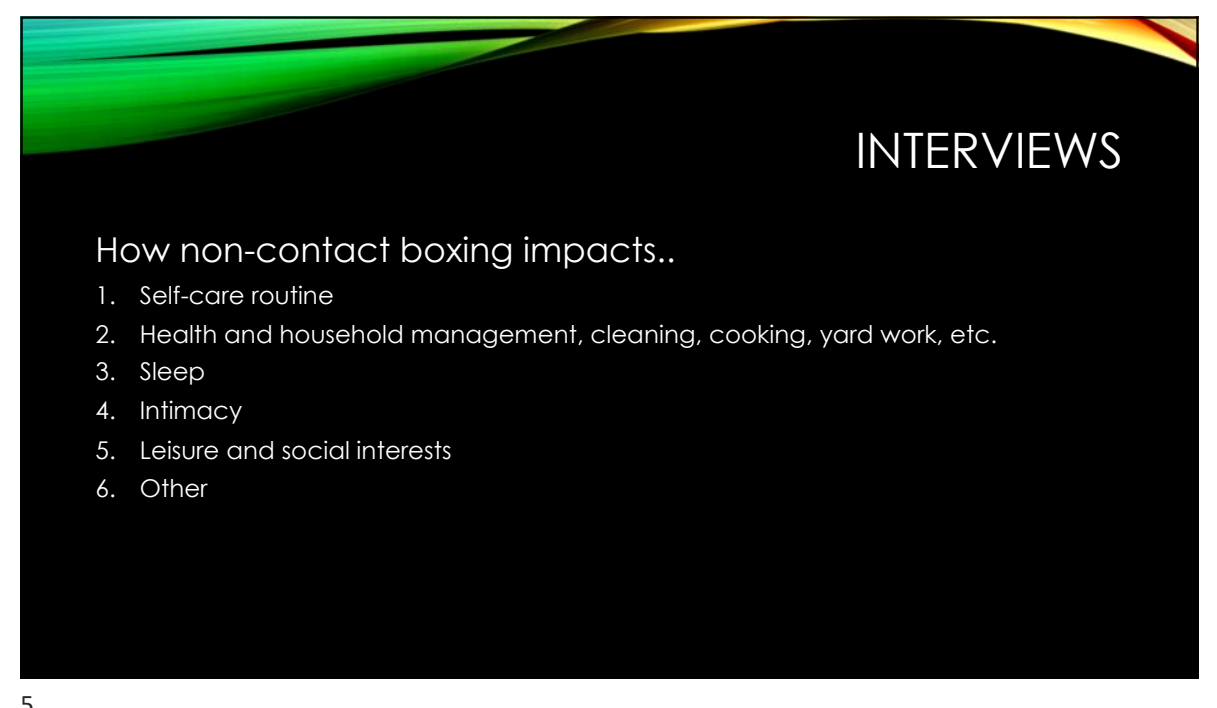

5

\section{RESULTS}




\section{THEMES}

1. Psychological Benefits

2. Sense of Community

3. Framework to Develop Routines

\section{PSYCHOLOGICAL BENEFITS}

"...I have a lot more confidence in my balance, in my strength than I did before Rock Steady. I can't imagine the difference really if I weren't in it."
"But if I go to Rock Steady... I'm good for the whole day. Energizes me. And like / said, then I forget that I'm having a bad day. It just clears up." - 


\section{SENSE OF COMMUNITY}

"I feel at home here. I feel like my friends are here. The people who are here understand me..."
"Some of our closest friends now are here at Rock Steady."
"They're your family, they're fun, fun to see and you joke around and do you have a good time and that makes a big difference in itself. Even without the exercise."

\section{FRAMEWORK TO DEVELOP ROUTINES}

"I believe I would be way worse condition if I wasn't doing Rock Steady... it's having a place to go to, having a regular schedule, the social part as well and stuff."
"The biggest impact is just overall physical strength and health and wellbeing, you know" 


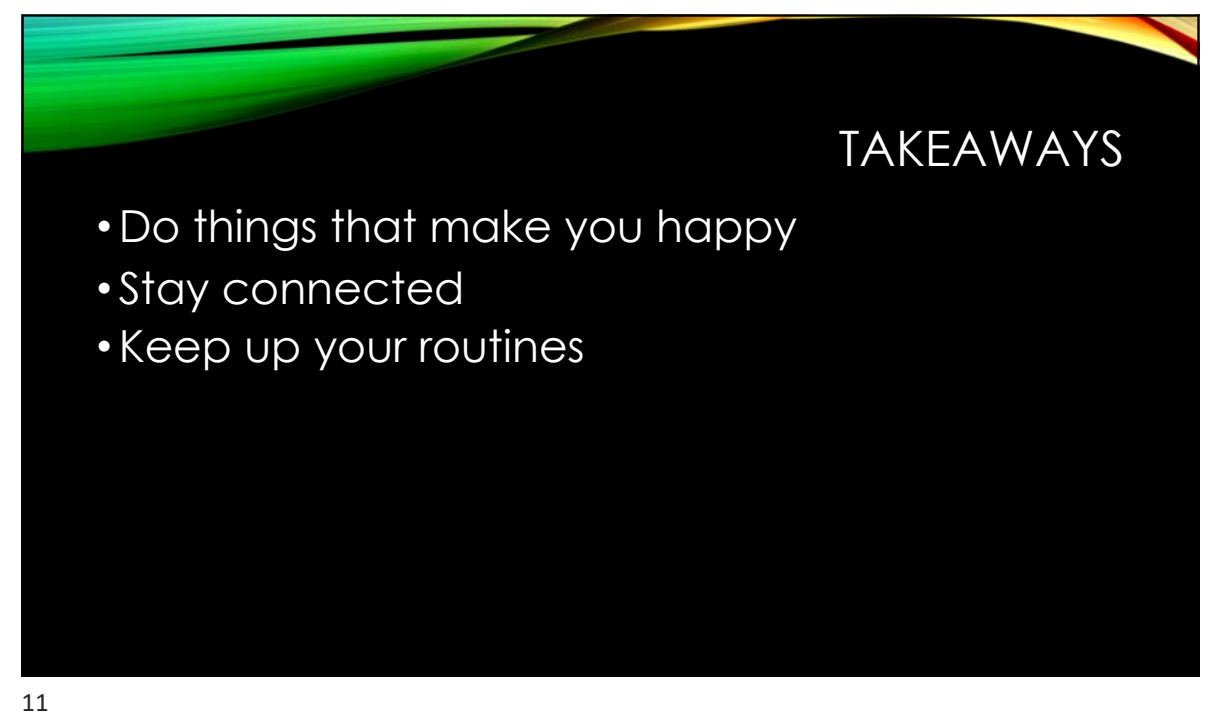

\section{CONTACT INFORMATION}

Please email me at k..bignal@usa.edu with any questions or comments!

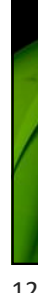

12 


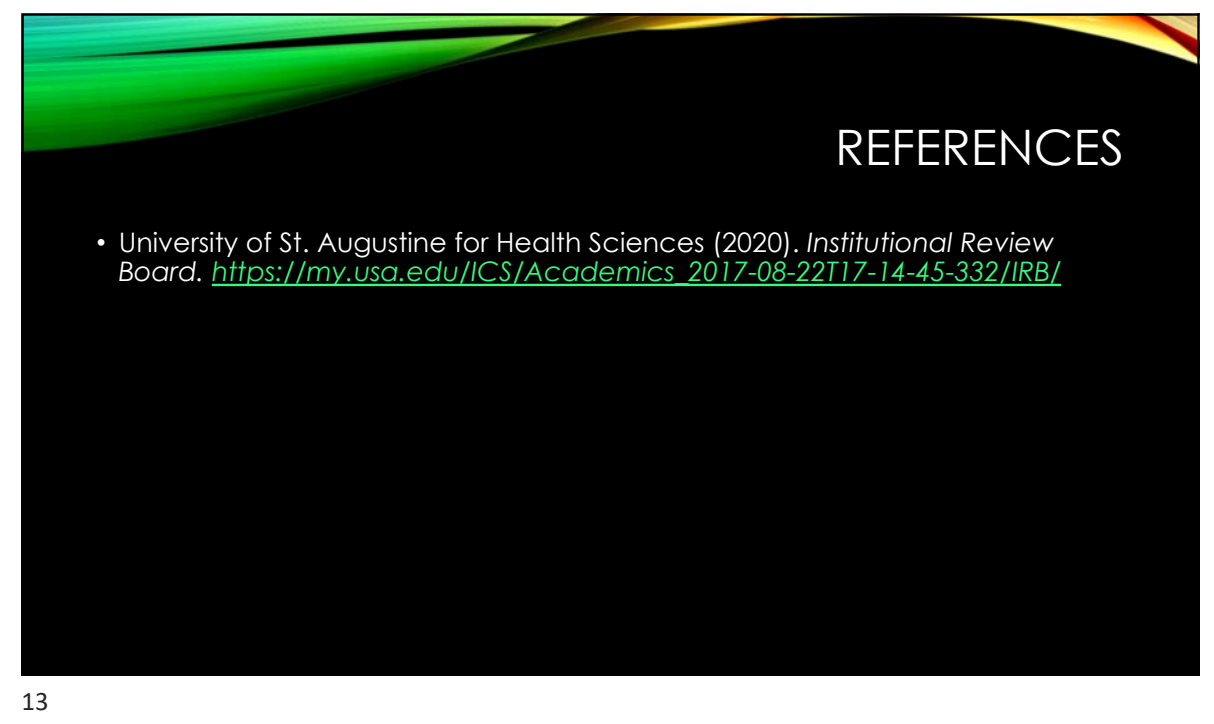




\section{Appendix I \\ Research Poster}

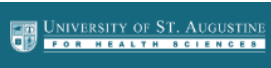

Doctor of Occupational Therapy Program

Perceived Impact of Non-contact Boxing on Daily Life and Occupational Participation at Home Among Individuals with Parkinson's Disease

Kristen Bignal; Susan MacDermott, OTD, OTR/L; Becki Cohill, OTD, OTR/L

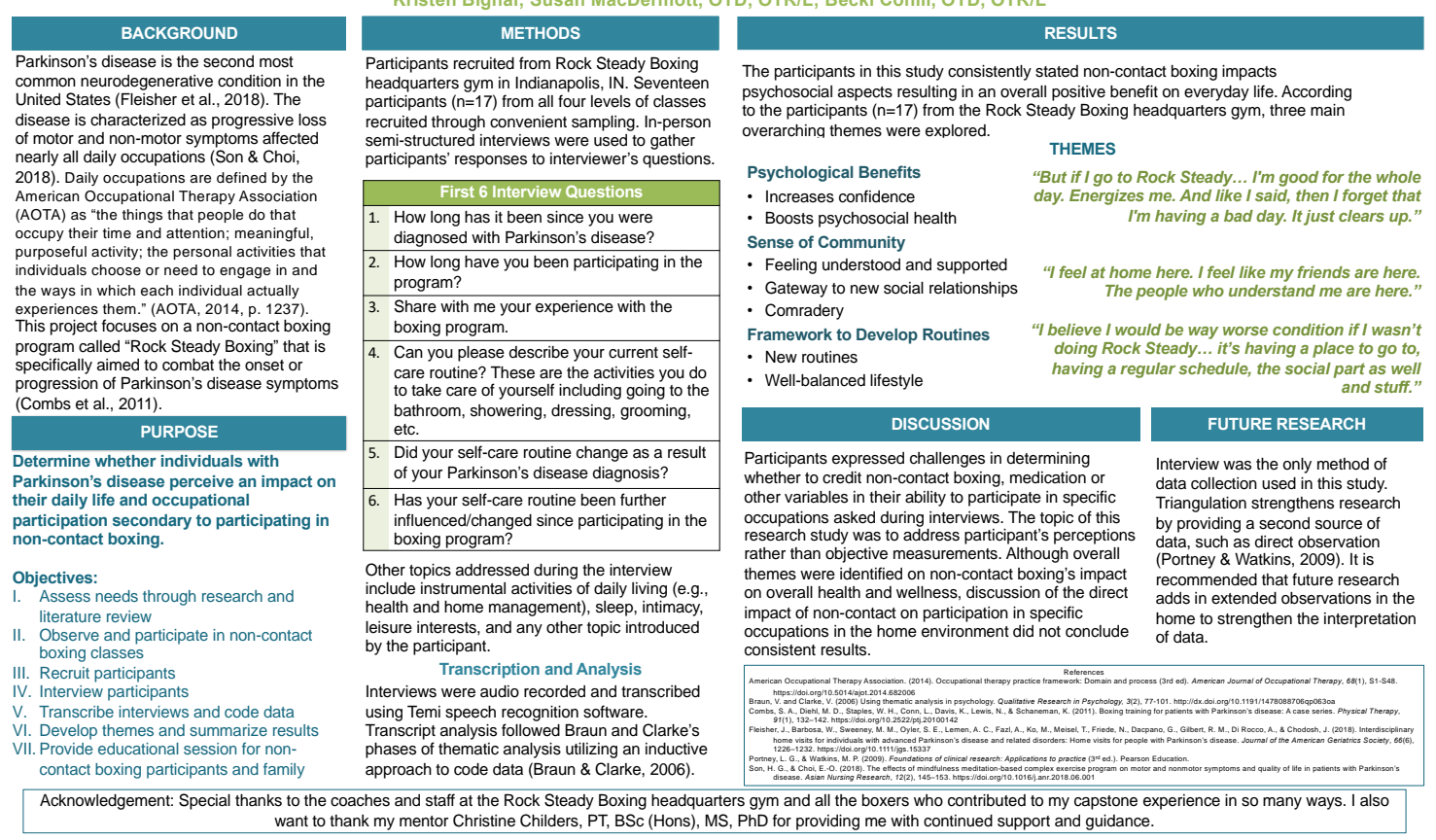




\section{Appendix J Journal Publication}

This research study, "Perceived Impact of Non-contact Boxing on Daily Life and Occupational Participation at Home Among Individuals with Parkinson's Disease" will tentatively be submitted to the Journal of Parkinson's Disease for publication. This journal seems most appropriate as they accept a variety of Parkinson's disease related research and targets an audience of basic science researchers and clinicians in the areas of Parkinson's disease and related disorders. Journal publication will be pursued further after completion of the student's capstone experience in efforts to add this research to the current literature to further advance knowledge and practice on related topics. 